Article

\title{
Hesitant Fuzzy Topological Spaces
}

\author{
Jeong-Gon Lee ${ }^{1, * \mathbb{D}}$ and Kul Hur ${ }^{2}$ \\ 1 Division of Applied Mathematics, Nanoscale Science and Technology Institute, Wonkwang University, \\ Iksan 54538, Korea \\ 2 Department of Applied Mathematics, Wonkwang University, 460, Iksan-daero, Iksan-Si, \\ Jeonbuk 54538, Korea; kulhur@wku.ac.kr \\ * Correspondence: jukolee@wku.ac.kr
}

Received: 1 January 2020; Accepted: 30 January 2020; Published: 4 February 2020

\begin{abstract}
In this study, we define a hesitant fuzzy topology and base, obtain some of their properties, respectively, and give some examples. Next, we introduce the concepts of a hesitant fuzzy neighborhood, Q-neighborhood, closure, and interior and obtain some of their properties, respectively. Furthermore, we define a hesitant fuzzy continuous mapping and investigate some of its properties. Furthermore, we define a hesitant fuzzy subspace and obtain some of its properties. In particular, we obtain the Pasting lemma. We investigate the concept of hesitant fuzzy product space and study some of its properties.
\end{abstract}

Keywords: hesitant fuzzy set; hesitant fuzzy topology; hesitant fuzzy bees; hesitant fuzzy neighborhood and Q-neighborhood; hesitant fuzzy closure; hesitant fuzzy interior; hesitant fuzzy continuous mapping; hesitant fuzzy subspace; hesitant fuzzy product space

\section{Introduction}

In 1965, Zadeh [1] introduced the concept of a fuzzy set as a generalization of a crisp set. Chang [2] defined initially the notion of fuzzy topological spaces, and then, many researchers [3-12] investigated various properties, for example neighborhood systems, Q-neighborhood systems, continuities, compactness, initial structures, and separation axioms in fuzzy topological spaces.

In 2010, Torra [13] introduced the notion of a hesitant fuzzy set as an extension of a fuzzy set proposed by Zadeh [1] $([14,15])$. In a hesitant fuzzy set, since the membership function takes values from the power set $[0,1]$ (see Definition 1), it helps in dealing with the situation effectively. Then, hesitant fuzzy set theory has many applications in various fields like decision making problems, decision support systems, clustering algorithms, algebras, etc. After that time, hesitant fuzzy set theory has been developed rapidly by some scholars in theory and practice.

Xia and Xu [16] applied a hesitant fuzzy set to decision making by defining "hesitant fuzzy information aggregation". Jun et al. [17] studied hesitant fuzzy bi-ideals in semigroups. Deepark and John [18] introduced a basic version of hesitant fuzzy rough sets through hesitant fuzzy relations. Furthermore, they studied the homomorphisms of hesitant fuzzy subgroups and hesitant fuzzy subrings and ideals in [19-21]. On the other hand, Jun and Ahn [22] applied hesitant fuzzy sets to $B C K / B C I$-algebras. Kim et al. [23] gave characterizations of a hesitant fuzzy positive implicative ideal, a hesitant fuzzy implicative ideal, and a hesitant fuzzy commutative ideal, respectively in $B C K$-algebras. Furthermore, they [24] introduced the category HSet $(H)$ consisting of all hesitant $H$-fuzzy spaces and all morphisms between them and studied HSet $(H)$ in the sense of a topological universe. Recently, Mathew et al. [25] defined a hesitant fuzzy topology and dealt with closures, interiors, continuities, connectedness, and compactness in hesitant fuzzy topological spaces.

The purpose of this paper is to investigate the topological properties on hesitant fuzzy sets. First, we define a hesitant fuzzy topology and base, obtain some of their properties, respectively, and give 
some examples. Second, we introduce the concepts of a hesitant fuzzy neighborhood, Q-neighborhood, closure, and interior and obtain some of their properties, respectively (in particular, see Propositions 6, 7, 9, and 10). Third, we define a hesitant fuzzy continuous mapping and investigate some of its properties. Fourth, we define a hesitant fuzzy subspace and obtain some of its properties. In particular, we obtain the Pasting lemma (see Proposition 31). Finally, we introduce the notion of a hesitant fuzzy product space and study some of its properties.

\section{Preliminaries}

In this section, we list some definitions and results needed in the later sections.

Definition 1. ([24]). Let $X$ be a reference set, and let $P[0,1]$ denote the power set of $[0,1]$. Then, a mapping $h: X \rightarrow P[0,1]$ is called a hesitant fuzzy set in $X$.

The hesitant fuzzy empty (resp. whole) set, denoted by $h^{\mathbf{0}}$ (resp. $h^{\mathbf{1}}$ ), is a hesitant fuzzy set in $X$ defined as: for each $x \in X$,

$$
h^{0}(x)=\phi\left[\operatorname{resp} . h^{\mathbf{1}}(x)=[0,1]\right]
$$

Especially, we will denote the set of all hesitant fuzzy sets in $X$ as $H S(X)$.

In fact, we can easily see that the hesitant fuzzy empty set and whole set are complete ignorance and a set of nonsense, respectively, introduced by Tora [13].

Definition 2. ([18]). Let $h_{1}, h_{2} \in H S(X)$. Then:

(i) we say that $h_{1}$ is a subset of $h_{2}$, denoted by $h_{1} \subset h_{2}$, if $h_{1}(x) \subset h_{2}(x)$, for each $x \in X$,

(ii) we say that $h_{1}$ is equal to $h_{2}$, denoted by $h_{1}=h_{2}$, if $h_{1} \subset h_{2}$ and $h_{2} \subset h_{1}$.

Definition 3. ([24]). Let $h_{1}, h_{2} \in H S(X)$, and let $\left(h_{j}\right)_{j \in J} \subset H S(X)$. Then:

(i) the intersection of $h_{1}$ and $h_{2}$, denoted by $h_{1} \widetilde{\cap} h_{2}$, is a hesitant fuzzy set in $X$ defined as follows: for each $x \in X$,

$$
\left(h_{1} \widetilde{\cap} h_{2}\right)(x)=h_{1}(x) \cap h_{2}(x),
$$

(ii) the intersection of $\left(h_{j}\right)_{j \in J}$, denoted by $\widetilde{\bigcap}_{j \in J} h_{j}$, is a hesitant fuzzy set in $X$ defined as follows: for each $x \in X$,

$$
\left(\widetilde{\bigcap_{j \in J}} h_{j}\right)(x)=\bigcap_{j \in J} h_{j}(x),
$$

(iii) the union of $h_{1}$ and $h_{2}$, denoted by $h_{1} \widetilde{\cup} h_{2}$, is a hesitant fuzzy set in $X$ defined as follows: for each $x \in X$,

$$
\left(h_{1} \widetilde{\cup} h_{2}\right)(x)=h_{1}(x) \cup h_{2}(x),
$$

(iv) the union of $\left(h_{j}\right)_{j \in J}$, denoted by $\widetilde{U}_{j \in J} h_{j}$, is a hesitant fuzzy set in $X$ defined as follows: for each $x \in X$,

$$
\left.\widetilde{\left(\bigcup_{j \in J}\right.} h_{j}\right)(x)=\bigcup_{j \in J} h_{j}(x) \text {. }
$$

Definition 4. ([24]). Let $X$ be a nonempty set, and let $h \in H S(X)$. Then, the complement of $h$, denoted by $h^{c}$, is a hesitant fuzzy set in $X$ defined as: for each $x \in X$,

$$
h^{c}(x)=h(x)^{c}=[0,1] \backslash h(x) .
$$

Result 1. ([24], Proposition 3.14). Let $X$ be a nonempty set; let $h, h_{1}, h_{2}, h_{3} \in H S(X) ;$ and let $\left(h_{j}\right)_{j \in J} \subset$ HS $(X)$. Then:

(1) (Idempotent laws): $h \widetilde{\cup} h=h, h \widetilde{\cap} h=h$,

(2) (Commutative laws): $h_{1} \widetilde{\cup} h_{2}=h_{2} \widetilde{\cup} h_{1}, h_{1} \widetilde{\cap} h_{2}=h_{2} \widetilde{\cap} h_{1}$,

(3) (Associative laws): $h_{1} \widetilde{\cup}\left(h_{2} \widetilde{\cup} h_{3}\right)=\left(h_{1} \widetilde{\cup} h_{2}\right) \widetilde{\cup} h_{3}, h_{1} \widetilde{\cap}\left(h_{2} \widetilde{\cap} h_{3}\right)=\left(h_{1} \widetilde{\cap} h_{2}\right) \widetilde{\cap} h_{3}$, 
(4) (Distributive laws): $h_{1} \widetilde{\cup}\left(h_{2} \widetilde{\cap} h_{3}\right)=\left(h_{1} \widetilde{\cup} h_{2}\right) \widetilde{\cap}\left(h_{1} \widetilde{\cup} h_{3}\right)$,

$$
h_{1} \widetilde{\cap}\left(h_{2} \widetilde{\cup} h_{3}\right)=\left(h_{1} \widetilde{\cap} h_{2}\right) \widetilde{\cup}\left(h_{1} \widetilde{\cap} h_{3}\right),
$$

(4) $^{\prime}$ (Generalized distributive laws): $h \widetilde{\cup}\left(\widetilde{\bigcap}_{j \in J} h_{j}\right)=\widetilde{\bigcap}_{j \in J}\left(h \widetilde{\cup} h_{j}\right)$,

$$
h \widetilde{\cap}\left(\widetilde{U}_{j \in J} h_{j}\right)=\widetilde{U}_{j \in J}\left(h \widetilde{\cap} h_{j}\right),
$$

(5) (Absorption laws): $h_{1} \widetilde{\cup}\left(h_{1} \widetilde{\cap} h_{2}\right)=h_{1}, h_{1} \widetilde{\cap}\left(h_{1} \widetilde{\cup} h_{2}\right)=h_{1}$.

(6) (DeMorgan's laws): $\left(h_{1} \widetilde{\cup} h_{2}\right)^{c}=h_{1}^{c} \widetilde{\cap} h_{2}^{c},\left(h_{1} \widetilde{\cap} h_{2}\right)^{c}=h_{1}^{c} \widetilde{\cup} h_{2}^{c}$,

(6)' (Generalized DeMorgan's laws): $\left(\widetilde{U}_{j \in J} h_{j}\right)^{c}=\widetilde{\cap}_{j \in J} h_{j}^{c},\left(\widetilde{\bigcap}_{j \in J} h_{j}\right)^{c}=\widetilde{U}_{j \in J} h_{j}^{c}$

(7) $\left(h^{c}\right)^{c}=h$,

(8) $h_{1} \widetilde{\cap} h_{2} \subseteq h_{1}$ and $h_{2} \widetilde{\cap} h_{1} \subseteq h_{2}$,

(9) $h_{1} \subseteq h_{1} \widetilde{\cup} h_{2}$ and $h_{2} \subseteq h_{1} \widetilde{\cup} h_{2}$,

(10) if $h_{1} \subseteq h_{2}$ and $h_{2} \subseteq h_{3}$, then $h_{1} \subseteq h_{3}$,

(11) if $h_{1} \subseteq h_{2}$, then $h_{1} \widetilde{\cap} h \subseteq h_{2} \widetilde{\cap} h$ and $h_{1} \widetilde{\cup} h \subseteq h_{2} \widetilde{\cup} h$,

(12) $h^{\mathbf{0}} \subseteq h \subseteq h^{\mathbf{1}}$,

(13) $h \widetilde{\cap} h^{\mathbf{0}}=h^{\mathbf{0}}, h \widetilde{\cup} h^{\mathbf{0}}=h, h \widetilde{\cap} h^{\mathbf{1}}=h, h \widetilde{\cup} h^{\mathbf{1}}=h^{\mathbf{1}}$.

We can see that Kim et al. [24] redefined the intersection, the union, and the complement introduced by Tora [13] so that $\left(H S(X), \widetilde{\cap}, \widetilde{\cup}^{c}\right)$ is a Boolean algebra with the least element $h^{0}$ and the largest element $h^{1}$.

Definition 5. ([24]). Let $X$ and $Y$ be a nonempty sets; let $h_{X} \in H S(X)$ and $\left.h_{Y} \in H S(Y)\right)$; and let $f: X \rightarrow Y$ be a mapping. Then:

(i) the image of $h_{X}$ under $f$, denoted by $f\left(h_{X}\right)$, is a hesitant fuzzy set in $Y$ defined as follows: for each $y \in Y$,

$$
f\left(h_{X}\right)(y)= \begin{cases}\widetilde{U}_{x \in f^{-1}(y)} h_{X}(x) \text { if } f^{-1}(y) \neq \phi \\ \phi & \text { otherwise, }\end{cases}
$$

(ii) the preimage of $h_{Y}$ under $f$, denoted by $f^{-1}\left(h_{Y}\right)$, is a hesitant fuzzy set in $Y$ defined as follows: for each $x \in X$,

$$
f^{-1}\left(h_{Y}\right)(x)=h_{Y} \circ f(x) .
$$

Result 2. ([24], Proposition 3.16). Let $f: X \rightarrow Y$ be a mapping, and let $h_{X}, h_{X 1}, h_{X 2} \in H S(X)$, $\left(h_{X_{j}}\right)_{j \in J} \subset H S(X), h_{Y}, h_{Y 1}, h_{Y 2} \in H S(Y)$ and $\left(h_{Y_{j}}\right)_{j \in J} \subset H S(Y)$. Then:

(1) if $h_{X 1} \subseteq h_{X 2}$, then $f\left(h_{X 1}\right) \subseteq f\left(h_{X 2}\right)$,

(2) $f\left(h_{X 1} \widetilde{\cup} h_{X 2}\right)=f\left(h_{X 1}\right) \widetilde{\cup} f\left(h_{X 2}\right), f\left(\widetilde{\cup}_{j \in J} h_{X_{j}}\right)=\widetilde{\cup}_{j \in J} f\left(h_{X_{j}}\right)$,

(3) $f\left(h_{X 1} \widetilde{\cap} h_{X 2}\right) \subseteq f\left(h_{X 1}\right) \widetilde{\cap} f\left(h_{X 2}\right), f\left(\widetilde{\cap}_{j \in J} h_{X_{j}}\right) \subseteq \widetilde{\cap}_{j \in J} f\left(h_{X_{j}}\right)$,

(3)' if $f$ is injective, then $f\left(h_{X 1} \widetilde{\cap} h_{X 2}\right)=f\left(h_{X 1}\right) \widetilde{\cap} f\left(h_{X 2}\right), f\left(\widetilde{\cap}_{j \in J} h_{X_{j}}\right)=\widetilde{\bigcap}_{j \in J} f\left(h_{X_{j}}\right)$,

(4) $f(A)=h^{0}$ if and only if $A=h^{0}$,

(5) if $h_{Y 1} \subseteq h_{\curlyvee 2}$, then $f^{-1}\left(h_{Y 1}\right) \subseteq f^{-1}\left(h_{Y 2}\right)$,

(6) $f^{-1}\left(h_{Y 1} \widetilde{\cup} h_{{ }^{2} 2}\right)=f^{-1}\left(h_{\curlyvee 1}\right) \widetilde{\cup} f^{-1}\left(h_{Y 2}\right), f^{-1}\left(\widetilde{\cup}_{j \in J} h_{Y_{j}}\right)=\widetilde{U}_{j \in J} f^{-1}\left(h_{Y_{j}}\right)$,

(7) $f^{-1}\left(h_{Y 1} \widetilde{\cap} h_{Y 2}\right) \subseteq f^{-1}\left(h_{Y 1}\right) \widetilde{\cap} f^{-1}\left(h_{Y 2}\right), f^{-1}\left(\widetilde{\cap}_{j \in J} h_{Y_{j}}\right) \subseteq \widetilde{\cap}_{j \in J} f^{-1}\left(h_{Y_{j}}\right)$,

(8) $f^{-1}\left(h_{Y}\right)=h^{\mathbf{1}}$ if and only if $h_{Y} \widetilde{\cap} f\left(h^{\mathbf{1}}\right)=h^{\mathbf{1}}$,

(9) $h_{X} \subset f^{-1} \circ f\left(h_{X}\right)$; in particular, $h_{X}=f^{-1} \circ f\left(h_{X}\right)$, if $f$ is injective,

(10) $f \circ f^{-1}\left(h_{Y}\right) \subset h_{Y}$; in particular, $f \circ f^{-1}\left(h_{Y}\right)=h_{Y}$, if $f$ is surjective.

Definition 6. ([26]). Let $h \in H S(X)$. Then, $h$ is called a hesitant fuzzy point with the support $x \in X$ and the value $\lambda$, denoted by $x_{\lambda}$, if $x_{\lambda}: X \rightarrow P[0,1]$ is the mapping given by: for each $y \in X$,

$$
x_{\lambda}(y)=\left\{\begin{array}{lr}
\lambda \subset[0,1] & \text { if } y=x \\
\phi & \text { otherwise. }
\end{array}\right.
$$


In particular, $H_{P}(X)$ is called the set of all hesitant fuzzy points in $X$.

Definition 7. ([26]). Let $h \in H S(X)$, and let $x_{\lambda} \in H_{P}(X)$. Then, $x_{\lambda}$ is said to belong to $h$, denoted by $x_{\lambda} \in h$, if $\lambda \subset h(x)$.

It is obvious that $h=\widetilde{U}_{x_{\lambda} \in h} x_{\lambda}$.

Result 3. ([26], Theorem 3.3). Let $h_{1}, h_{2} \in H S(X)$, and let $\left(h_{j}\right)_{j \in J} \subset H S(X)$. Then:

(1) $h_{1} \subset h_{2}$ if and only if $x_{\lambda} \in h_{2}$, for each $x_{\lambda} \in h_{1}$.

(2) $x_{\lambda} \in h_{1} \widetilde{\cap} h_{2}$ if and only if $x_{\lambda} \in h_{1}$ and $x_{\lambda} \in h_{2}$.

(3) If $x_{\lambda} \in h_{1}$ or $x_{\lambda} \in h_{2}$, then $x_{\lambda} \in h_{1} \widetilde{\cup} h_{2}$.

(4) $x_{\lambda} \in \widetilde{\bigcap}_{j \in J} h_{j}$ if and only if $x_{\lambda} \in h_{j}$, for each $j \in J$.

(5) If $x_{\lambda} \in h_{j}$ for some $j \in J$, then $x_{\lambda} \in \widetilde{U}_{j \in J} h_{j}$.

Remark 1. ([26], Theorem 3.3). From Definitions 2.6 and 2.9, we can easily see that for any mapping $f: X \rightarrow Y$ and each $x_{\lambda} \in H_{P}(X), f\left(x_{\lambda}\right)=f(x)_{\lambda}$

\section{Topology of Hesitant Fuzzy Sets}

A fuzzy topology is a concept that combines order structure and topology. Chang [2] studied fuzzy topological spaces in the sense of point-like structure. Ekici [3] showed that the concept of fuzzy topology may be relevant to quantum particle physics in connection with string theory. Silva and Landim [4] showed that the notion of a fuzzy space topology can be used to explain the origin of the black hole entropy. Since a hesitant fuzzy topology is a more flexible variation of a fuzzy topology, we expect that a hesitant fuzzy topology may be more effectively applicable.

In this section, we introduce the concepts of hesitant fuzzy topologies, bases, and sub-bases in hesitant fuzzy topological spaces, find some of their basic properties, and give examples. One can easily see that our definition of a hesitant fuzzy topology is different from the one introduced by Mathew et al. [25]. Moreover, in order to apply hesitant fuzzy sets to a topology, we refer mainly to $[27,28]$.

Definition 8. Let $X$ be a nonempty set, and let $\tau \subset H S(X)$. Then, $\tau$ is called a hesitant topology $(H F T)$ on $X$, if it satisfies the following axioms:

(HFT1) $h^{\mathbf{0}}, h^{\mathbf{1}} \in \tau$,

(HFT2) $h_{1} \widetilde{\cap} h_{2} \in \tau$, for any $h_{1}, h_{2} \in \tau$,

(HFT3) $\widetilde{U}_{j \in J} h_{j} \in \tau$, for each $\left(h_{j}\right)_{j \in J} \subset \tau$.

Especially, the pair $(X, \tau)$ is called a hesitant fuzzy topological space. Each member of $\tau$ is called a hesitant fuzzy open set (HFOS) in X. A hesitant fuzzy set h in X is called a hesitant fuzzy closed set (HFCS) in $(X, \tau)$, if $h^{c} \in \tau$.

We will denote the set of all HFTson X (resp. HFOSsand HFCSsin X) as HFT (X) (resp. HFO (X) and $\operatorname{HFC}(X))$

Example 1. (1) Let $X=\{a, b, c\}$, and consider hesitant fuzzy sets in $X$ given by:

$$
\begin{aligned}
& h_{1}(a)=[0.8,1], h_{1}(b)=\{0.3,0.6,0.9\}, h_{1}(c)=[0.8,1), \\
& h_{2}(a)=[0.6,1), h_{2}(b)=\{0.3,0.6,0.8\}, h_{2}(c)=(0.8,1], \\
& h_{3}(a)=[0.8,1), h_{3}(b)=\{0.3,0.6\}, h_{3}(c)=(0.8,1), \\
& h_{4}(a)=[0.8,1], h_{4}(b)=\{0.3,0.6,0.8,0.9\}, h_{4}(c)=[0.8,1] .
\end{aligned}
$$

Then, we can easily see that $\tau=\left\{h^{\mathbf{0}}, h^{\mathbf{1}}, h_{1}, h_{2}, h_{3}, h_{4}\right\} \in \operatorname{HFT}(X)$.

(2) Let $X$ be a nonempty set. Then, clearly, the class $\left\{h^{\mathbf{0}}, h^{\mathbf{1}}\right\} \in \operatorname{HFT}(X)$. In particular, $\left\{h^{\mathbf{0}}, h^{\mathbf{1}}\right\}$ will be called a hesitant fuzzy indiscrete topology on $X$ and denoted by $\tau_{\mathbf{0}}$. The pair $\left(X, \tau_{\mathbf{0}}\right)$ is called a hesitant fuzzy indiscrete space. 
(3) Let $X$ be a nonempty set. Then, we can easily see that the class $H S(X)$ is a hesitant fuzzy topology on X. In particular, HS $(X)$ will be called a hesitant fuzzy discrete topology on $X$ and denoted by $\tau_{1}$. The pair $\left(X, \tau_{\mathbf{1}}\right)$ is called a hesitant fuzzy discrete space.

From Definition 8, we have the following result.

Proposition 1. Let $(X, \tau)$ be a hesitant fuzzy topological space. Then:

(1) $h^{\mathbf{0}}, h^{\mathbf{1}} \in \operatorname{HFC}(X)$,

(2) $h_{1} \widetilde{\cup} h_{2} \in H F C(X)$, for any $h_{1}, h_{2} \in H F C(X)$,

(2) $\widetilde{\cap}_{j \in J} h_{j} \in \operatorname{HFC}(X)$, for each $\left(h_{j}\right)_{j \in J} \subset \operatorname{HFC}(X)$.

Proposition 2. If $\left(\tau_{j}\right)_{j \in J} \subset \operatorname{HFT}(X)$, then $\widetilde{\bigcap}_{j \in J} \tau_{j} \in \operatorname{HFT}(X)$.

Proof. Let $\tau=\bigcap_{j \in J} \tau_{j}$. Then, clearly, $h^{\mathbf{0}}, h^{\mathbf{1}} \in \tau_{j}$, for each $j \in J$. Thus, $h^{0}, h^{\mathbf{1}} \in \bigcap_{j \in J} \tau_{j}$. Therefore, $h^{0}, h^{\mathbf{1}} \in \tau$. Hence, $\tau$ satisfies Axiom (HFT1).

Let $h_{1}, h_{2} \in \tau$. Then, $h_{1}, h_{2} \in \tau_{j}$, for each $j \in J$. Thus, $h_{1} \widetilde{\cap} h_{2} \in \tau_{j}$, for each $j \in J$. Therefore, $h_{1} \widetilde{\cap} h_{2} \in \bigcap_{j \in J} \tau_{j}$, i.e., $h_{1} \widetilde{\cap} h_{2} \in \tau$. Hence, $\tau$ satisfies the axiom (HFT2).

Now, let $\left(h_{k}\right)_{k \in K} \subset \tau$. Then, $\left(h_{k}\right)_{k \in K} \subset \tau_{j}$, for each $j \in J$. Therefore, $\widetilde{U}_{k \in K} h_{k} \in \bigcap_{j \in J} \tau_{j}$, i.e., $\widetilde{U}_{k \in K} h_{k} \in \tau$. Hence, $\tau$ satisfies Axiom (HFT3). This completes the proof.

For any $\tau_{1}, \tau_{2} \in \operatorname{HFT}(X)$, we say that $\tau_{1}$ is weaker or coarser than $\tau_{2}$, if $\tau_{1} \subset \tau_{2}$. In this case, we say that $\tau_{2}$ is stronger or finer than $\tau_{1}$.

The following is the immediate result of Example 1 and Proposition 2.

Proposition 3. Let $\left(\tau_{j}\right)_{j \in J} \subset \operatorname{HFT}(X)$. Then, it forms a complete lattice with respect to the set inclusion relation of which $\tau_{\mathbf{0}}$ is the smallest element and $\tau_{\mathbf{1}}$ is the largest element.

Definition 9. Let $(X, \tau)$ be a hesitant fuzzy topological space, and let $\mathcal{B} \subset \tau$. Then, $\mathcal{B}$ is called a base for $\tau$, if for each $h \in \tau, h=h^{0}$, or there is $\mathcal{B}^{\prime} \subset \mathcal{B}$ such that $h=\widetilde{\cup} \mathcal{B}^{\prime}$.

Example 2. (1) Let $X$ be a nonempty set. Then, $H_{P}(X)$ is a base for $\tau_{\mathbf{1}}$.

(2) Let $X=\{a, b, c\}$, and consider $\mathcal{B}=\left\{h_{1}, h_{2}, h^{\mathbf{1}}\right\}$,

where $h_{1}(a)=\{0.6\} \cup[0.7,1), h_{1}(b)=\{0.4,0.6\}, h_{1}(c)=[0,0.6) \cup\{0.8\}$,

$h_{2}(a)=\{0.3\} \cup(0.8,1], h_{2}(b)=\{0.6,0.8\}, h_{2}(c)=(0.5,0.6] \cup\{0.7\}$.

Suppose $\mathcal{B}$ is a base for an HFT $\tau$ on X. Then, by the definition of a base, $\mathcal{B} \subset \tau$. Since $h_{1}, h_{2} \in \tau$, $h_{1} \widetilde{\cap} h_{2} \in \tau$. It is clear that $h_{1} \widetilde{\cap} h_{2} \neq h^{0}$. However, for any $\mathcal{B}^{\prime} \subset \mathcal{B}, h_{1} \widetilde{\cap} h_{2} \neq \widetilde{\cup} \mathcal{B}^{\prime}$. Thus, by Definition $9, \mathcal{B}$ is not a base for some hesitant fuzzy topology on $X$.

Theorem 1. Let $X$ be a nonempty set. Then, $\mathcal{B} \subset H S(X)$ is a base for some HFT on $X$ if and only if it satisfies the following conditions:

(1) $h^{\mathbf{1}}=\widetilde{\cup} \mathcal{B}$,

(2) if $B_{1}, B_{2} \in \mathcal{B}$ and $x_{\lambda} \in B_{1} \widetilde{\cap} B_{2}$, then there is $B \in \mathcal{B}$ such that:

$$
x_{\lambda} \in B \text { and } B \subset B_{1} \widetilde{\cap} B_{2} .
$$

Proof. Suppose $\mathcal{B} \subset H S(X)$ is a base for some HFT $\tau$ on $X$. Since $h^{\mathbf{1}} \in \tau$, it is clear that $h^{\mathbf{1}}=\widetilde{\cup} \mathcal{B}$. Then, the condition (1) is satisfied.

Now, suppose $B_{1}, B_{2} \in \mathcal{B}$ and $x_{\lambda} \in B_{1} \widetilde{\cap} B_{2}$. Then, clearly, $B_{1}, B_{2} \in \tau$. Thus, $B_{1} \widetilde{\cap} B_{2} \in \tau$ and $B_{1} \widetilde{\cap} B_{2} \neq h^{0}$. Since $\mathcal{B}$ is a base for $\tau$, there is $\mathcal{B}^{\prime} \subset \mathcal{B}$ such that $B_{1} \widetilde{\cap} B_{2}=\widetilde{\cup} \mathcal{B}^{\prime}$. Since $x_{\lambda} \in B_{1} \widetilde{\cap} B_{2}$, there is $B \in \mathcal{B}^{\prime}$ such that:

$$
x_{\lambda} \in B \text { and } B \subset B_{1} \widetilde{\cap} B_{2} .
$$


Therefore, Condition (2) is satisfied.

Conversely, suppose the necessary conditions hold, and let:

$$
\tau=\left\{h^{0}\right\} \cup\left\{h: h=\widetilde{\cup} \mathcal{B}^{\prime}, \text { for some } \mathcal{B}^{\prime} \subset \mathcal{B}\right\} .
$$

Then, clearly, $h^{\mathbf{0}}, h^{\mathbf{1}} \in \tau$. Furthermore, we can easily see that $\tau$ is closed under arbitrary union. Thus, $\tau$ satisfies Axioms (HFT1) and (HFT3).

Let $h_{1}, h_{2} \in \tau$, and let $x_{\lambda} \in h_{1} \widetilde{\cap} h_{2}$. Then, by the definition of $\tau$, there are $B_{1}, B_{2} \in \mathcal{B}$ such that $x_{\lambda} \in B_{1} \subset h_{1}$ and $x_{\lambda} \in B_{2} \subset h_{2}$. Thus, by Condition (2), there is $B \in \mathcal{B}$ such that $x_{\lambda} \in B \subset B_{1} \widetilde{\cap} B_{2}$. Therefore, $h_{1} \widetilde{\cap} h_{2}=\widetilde{\cup} \mathcal{B}^{\prime}$, for some $\mathcal{B}^{\prime} \subset \mathcal{B}$. Hence, $h_{1} \widetilde{\cap} h_{2} \in \tau$. Therefore, $\tau$ satisfies Axiom (HFT2). This completes the proof.

Definition 10. If $\mathcal{B}$ is a base for an HFT $\tau$ on $X$, then $\tau$ is called the hesitant fuzzy topology generated by $\mathcal{B}$. In fact,

$$
\tau=\left\{h^{0}\right\} \cup\left\{h: h=\widetilde{\cup} \mathcal{B}^{\prime}, \text { for some } \mathcal{B}^{\prime} \subset \mathcal{B}\right\} .
$$

Example 3. Let $X=\{a, b, c\}$, and consider $\mathcal{B}=\left\{h_{1}, h_{2}, h_{3}\right\}$,

where $h_{1}(a)=\{0.6\} \cup(0.7,1], h_{1}(b)=\{0\} \cup(0.6,1], h_{1}(c)=[0,0.6)$,

$$
\begin{aligned}
& h_{2}(a)=[0,0.8] \cup\{0.9\}, h_{2}(b)=(0,0.7) \cup\{0.8\}, h_{2}(c)=\{0.3\} \cup(0.5,1], \\
& h_{3}(a)=\{0.6,0.9\} \cup(0.7,0.8], h_{3}(b)=(0.6,0.7) \cup\{0.8\}, \\
& h_{3}(c)=\{0.3\} \cup(0.5,0.6) .
\end{aligned}
$$

Then, clearly, $h_{1} \widetilde{\cup} h_{2} \widetilde{\cup} h_{3}=h^{1}$. Furthermore, we can easily show that $\mathcal{B}$ satisfies Condition (2) of Theorem 1. Thus, $\mathcal{B}$ is a base for some HFT $\tau$ on X. In fact,

$$
\tau=\left\{h^{0}, h^{\mathbf{1}}, h_{1}, h_{2}, h_{3}\right\} .
$$

Definition 11. Let $\tau_{1}, \tau_{2} \in H F T(X)$, and let $\mathcal{B}_{1}$ and $\mathcal{B}_{2}$ be bases for $\tau_{1}$ and $\tau_{2}$, respectively. Then, $\mathcal{B}_{1}$ and $\mathcal{B}_{2}$ are said to be equivalent, if $\tau_{1}=\tau_{2}$.

Theorem 2. Let $\tau_{1}, \tau_{2} \in H F T(X)$, and let $\mathcal{B}_{1}$ and $\mathcal{B}_{2}$ be bases for $\tau_{1}$ and $\tau_{2}$, respectively. Then, the following are equivalent:

(1) $\tau_{2}$ is finer than $\tau_{1}$, i.e., $\tau_{1} \subset \tau_{2}$,

(2) for each $x_{\lambda} \in H_{P}(X)$ and each $B_{1} \in \mathcal{B}_{1}$ such that $x_{\lambda} \in B_{1}$, there is $B_{2} \in \mathcal{B}_{2}$ such that $x_{\lambda} \in B_{2} \subset B_{1}$.

Proof. (1) $\Rightarrow(2)$ : Suppose $\tau_{1} \subset \tau_{2}$; let $x_{\lambda} \in H_{P}(X)$, and let $B_{1} \in \mathcal{B}_{1}$ such that $x_{\lambda} \in B_{1}$. Then, clearly, $B_{1} \in \tau_{2}$. Since $\tau_{2}$ is generated by $\mathcal{B}_{2}$ and $x_{\lambda} \in B_{1}$, there is $B_{2} \in \mathcal{B}_{2}$ such that $x_{\lambda} \in B_{2} \subset B_{1}$.

(2) $\Rightarrow(1)$ : Suppose the necessary condition holds; let $h \in \tau_{1}$, and let $x_{\lambda} \in h$. Since $\tau_{1}$ is generated by $\mathcal{B}_{1}$, there is $B_{1} \in \mathcal{B}_{1}$ such that $x_{\lambda} \in B_{1} \subset h$. Then, by Condition (2), there is $B_{2} \in \mathcal{B}_{2}$ such that $x_{\lambda} \in B_{2} \subset B_{1}$. Thus, $B_{2} \subset h$. Therefore, $h=\widetilde{\cup} \mathcal{B}_{2}^{\prime}$, for some $\mathcal{B}_{2}^{\prime} \subset \mathcal{B}$. Hence, $h \in \tau_{2}$. Therefore, $\tau_{1} \subset \tau_{2}$.

Proposition 4. Let $(X, \tau)$ be a hesitant fuzzy topological space. Assume $\mathcal{B} \subset \tau$ such that for each $x_{\lambda} \in H_{P}(X)$ and each $U \in \tau$ with $x_{\lambda} \in U$, there is $B \in \mathcal{B}$ such that $x_{\lambda} \in B \subset U$. Then, $\mathcal{B}$ is a base for $\tau$.

Proof. Let $x_{\lambda} \in H_{P}(X)$. Since $h^{\mathbf{1}} \in \tau$, there is $B \in \mathcal{B}$ such that $\left.x_{\lambda} \in B\right]$ subseth $^{\mathbf{1}}$. Then, $h^{\mathbf{1}}=\widetilde{\cup} \mathcal{B}$.

Suppose $B_{1}, B_{2} \in \mathcal{B}$ and $x_{\lambda} \in B_{1} \widetilde{\cap} B_{2}$. Since $B_{1}, B_{2} \in \tau, B_{1} \widetilde{\cap} B_{2} \in \tau$. Then, there is $B \in \mathcal{B}$ such that $x_{\lambda} \in B \subset B_{1} \widetilde{\cap} B_{2}$. Thus, by Theorem $1, \mathcal{B}$ is a base for some HFT $\tau^{\prime}$ on $X$. By Theorem $2, \tau^{\prime}$ is finer than $\tau$. Since $\mathcal{B} \subset \tau$, arbitrary unions of members of $\mathcal{B}$ are members of $\tau$. Therefore, $\tau^{\prime} \subset \tau$. Hence, $\tau^{\prime}=\tau$. Therefore, $\mathcal{B}$ is a base for $\tau$. 
Definition 12. Let $(X, \tau)$ be a hesitant fuzzy topological space, and let $\mathcal{S} \subset \tau$. Then, $\mathcal{S}$ is called a sub-base for $\tau$, if the family:

$$
\left\{h \in H S(X): h=\widetilde{\cap}_{i=1}^{n} S_{i}, S_{i} \in \mathcal{S} \text { for } i=1,2, \cdots, n\right\}
$$

is a base for $\tau$.

Proposition 5. Let $X$ be a nonempty set, and let $\mathcal{S} \subset H S(X)$ such that $h^{\mathbf{1}}=\tilde{\cup} \mathcal{S}$. Then, there is a unique $H F T \tau$ such that $\mathcal{S}$ is a sub-base for $\tau$.

Proof. Let $\mathcal{B}=\left\{B \in H S(X): B=\widetilde{\cap}_{i=1}^{n} S_{i}, S_{i} \in \mathcal{S}\right.$ for $\left.i=1,2, \cdots, n\right\}$, and let:

$$
\tau=\left\{h \in H S(X): h=h^{0} \text { or } h=\widetilde{\cup} \mathcal{B}^{\prime} \text { for some } \mathcal{B}^{\prime} \subset \mathcal{B}\right\}
$$

Since $h^{\mathbf{1}}=\widetilde{U} \mathcal{S}, h^{\mathbf{1}} \in \tau$. By the definition of $\tau, h^{\mathbf{1 0}} \in \tau$. Then, $\tau$ satisfies Axiom (HFT1).

Let $\left(h_{j}\right)_{j \in J} \subset \tau$. Then, for each $j \in J$, there is $\mathcal{B}_{j} \subset \mathcal{B}$ such that $h_{j}=\tilde{\cup} \mathcal{B}_{j}$. Thus, $\widetilde{\cup}_{j \in J} h_{j}=$ $\widetilde{\cup}_{j \in J}\left(\widetilde{\cup} \mathcal{B}_{j}\right)$. Therefore, $\widetilde{U}_{j \in J} h_{j} \in \tau$. Hence, $\tau$ satisfies Axiom (HFT3).

Now, let $h_{1}, h_{2} \in \tau$, and let $x_{\lambda} \in h_{1} \widetilde{\cap} h_{2}$. Then, there are $B_{1}, B_{2} \in \mathcal{B}$ such that $x_{\lambda} \in B_{1} \widetilde{\cap} B_{2}, B_{1} \subset h_{1}$ and $B_{2} \subset h_{2}$. Since $B_{1}$ and $B_{2}$ are finite intersection of members of $\mathcal{S}$, respectively, $B_{1}, B_{2} \in \mathcal{B}$. Thus, $h_{1} \widetilde{\cap} h_{2}=\widetilde{\cup} \mathcal{B}^{\prime}$, for some $\mathcal{B}^{\prime} \subset \mathcal{B}$. Therefore, $h_{1} \widetilde{\cap} h_{2} \in \tau$. Hence, $\tau$ satisfies Axiom (HFT2). Therefore, $\tau \in \operatorname{HFT}(X)$. Furthermore, we can easily show that $\tau$ is unique.

Example 4. Let $X=\{a, b, c\}$, and consider $\mathcal{S}=\left\{h_{1}, h_{2}, h_{3}\right\}$,

where $h_{1}(a)=\{0.6\} \cup(0.7,1], h_{1}(b)=\{0\} \cup(0.6,1], h_{1}(c)=(0,0.6)$,

$h_{2}(a)=[0,0.7), h_{2}(b)=(0,0.6) \cup\{0.8\}, h_{2}(c)=\{0.3\} \cup(0.5,1)$,

$h_{3}(a)=\{0.7\}, h_{3}(b)=(0.5,0.7), h_{3}(c)=\{0\} \cup(0.8,1]$.

Then, clearly, $\widetilde{\cup} \mathcal{S}=h_{1} \widetilde{\cup} h_{2} \widetilde{\cup} h_{3}=h^{\mathbf{1}}$. Thus, $\widetilde{\cup} \mathcal{S}$ is a sub-base for the HFT $\tau$ on $X$. Furthermore, $\mathcal{B}=\left\{h_{1}, h_{2}, h_{3}, h_{4}, h_{5}, h_{6}\right\}$ is a base for $\tau$,

where $h_{4}(a)=\{0.6\}, h_{4}(b)=\{0.8\}, h_{4}(c)=\{0.3\} \cup(0.5,0.6)$,

$h_{5}(a)=\phi, h_{5}(b)=(0.5,0.6), h_{5}(c)=(0.8,1)$,

$h_{6}(a)=\phi, h_{6}(b)=(0.6,0.7), h_{6}(c)=\phi$.

Therefore, $\tau=\left\{h^{\mathbf{0}}, h^{\mathbf{1}}, h_{1}, h_{2}, h_{3}, h_{4}, h_{5}, h_{6}, h_{7}, h_{8}, h_{9}, h_{10}, h_{11}, h_{12}, h_{13}, h_{14}, h_{15}, h_{16}\right\}$,

where $h_{7}=h_{1} \widetilde{\cup} h_{1}, h_{8}=h_{2} \widetilde{\cup} h_{3}, h_{9}=h_{1} \widetilde{\cup} h_{3}, h_{10}=h_{1} \widetilde{\cup} h_{5}$,

$h_{11}=h_{2} \widetilde{\cup} h_{9}, h_{12}=h_{3} \widetilde{\cup} h_{4}, h_{13}=h_{4} \widetilde{\cup} h_{5}, h_{14}=h_{4} \widetilde{\cup} h_{6}$,

$h_{15}=h_{5} \widetilde{\cup} h_{6}, h_{16}=h_{4} \widetilde{\cup} h_{5} \widetilde{\cup} h_{6}$.

\section{Hesitant Fuzzy Neighborhoods, Interiors, and Closures}

It is well known that a neighborhood system generates a topology in a classical topological space. Then, the definition of a hesitant fuzzy neighborhood is necessary.

In this section, we define a hesitant fuzzy neighborhood of a hesitant fuzzy point, a hesitant fuzzy $Q$-neighborhood of a hesitant fuzzy quasi-coincident point, the hesitant fuzzy closure, interior, and exterior, study some of their properties, and give some examples.

Definition 13. Let $(X, \tau)$ be a hesitant fuzzy topological space; let $x_{\lambda} \in H_{P}(X)$; and let $N \in H S(G)$. Then, $N$ is called a hesitant fuzzy neighborhood (HFN) of $x_{\lambda}$, if there is $U \in \tau$ such that $x_{\lambda} \in U \subset N$.

We will denote the set of all HFNs of $x_{\lambda}$ in $(X, \tau)$ as $\operatorname{HFN}\left(x_{\lambda}\right)$.

The following is the immediate result of Definition 13.

Theorem 3. Let $(X, \tau)$ be a hesitant fuzzy topological space, and let $U \in H S(X)$. Then, $U \in \tau$ if and only if it is an HFN of each of its HFP, i.e., for each $x_{\lambda} \in U, U \in \operatorname{HFN}\left(x_{\lambda}\right)$. 
From the following result, we can see that the hesitant fuzzy neighborhoods have the property of the classical neighborhoods.

Proposition 6. Let $(X, \tau)$ be a hesitant fuzzy topological space, and let $x_{\lambda} \in H_{P}(X)$. Then, $H F N\left(x_{\lambda}\right)$ has the following properties:

(HFN1) $x_{\lambda} \in N$, for each $N \in \operatorname{HFN}\left(x_{\lambda}\right)$,

(HFN2) if $N \in \operatorname{HFN}\left(x_{\lambda}\right)$, then $M \in \operatorname{HFN}\left(x_{\lambda}\right)$, for each $M \in H S(X)$ such that $N \subset M$,

(HFN3) if $N, M \in H F N\left(x_{\lambda}\right)$, then $N \widetilde{\cap} M \in \operatorname{HFN}\left(x_{\lambda}\right)$,

(HFN4) if $N \in \operatorname{HFN}\left(x_{\lambda}\right)$, then there is $M \in \operatorname{HFN}\left(x_{\lambda}\right)$ such that $N \in \operatorname{HFN}\left(y_{\mu}\right)$, for each $y_{\mu} \in M$.

Proof. (1) The proof is clear.

(2) Suppose $N \in \operatorname{HFN}\left(x_{\lambda}\right)$, and let $N \widetilde{\cap} M \in H F N\left(x_{\lambda}\right)$. Then, there is $U \in \tau$ such that $x_{\lambda} \in U \subset$ $N$. Thus, $x_{\lambda} \in U \subset M$. Therefore, $M \in \operatorname{HFN}\left(x_{\lambda}\right)$.

(3) Suppose $N, M \in H F N\left(x_{\lambda}\right)$. Then, there are $U, V \in \tau$ such that $x_{\lambda} \in U \subset N$ and $x_{\lambda} \in V \subset M$. Thus, $x_{\lambda} \in U \widetilde{\cap} V \subset N \widetilde{\cap} M$ and $U \widetilde{\cap} V \in \tau$. Therefore, $N \widetilde{\cap} M \in \operatorname{HFN}\left(x_{\lambda}\right)$.

(4) Suppose $N \in \operatorname{HFN}\left(x_{\lambda}\right)$. Then, there is $M \in \tau$ such that $x_{\lambda} \in M \subset N$. Since $M \in \tau$, by Theorem 3, $M \in H F N\left(y_{\mu}\right)$, for each $y_{\mu} \in M$. Thus, for each $y_{\mu} \in M$, there is $V \in \tau$ such that $y_{\mu} \in V \subset M \subset N$. Therefore, $N \in \operatorname{HFN}\left(y_{\mu}\right)$. This completes the proof.

Proposition 7. Let $X$ be a nonempty set, and let for each $x_{\lambda} \in H_{P}(X)$ there be a nonempty collection $\mathcal{B}\left(x_{\lambda}\right)$ of hesitant fuzzy sets in X satisfying Properties (HFN1)-(HFN4). Then, there is a unique HFT $\tau$ on $X$ such that for each $x_{\lambda} \in H_{P}(X), \mathcal{B}\left(x_{\lambda}\right)=H_{F N}\left(x_{\lambda}\right)$, where $H F N_{\tau}\left(x_{\lambda}\right)$ denotes the set of all HFNs of $x_{\lambda}$ in $(X . \tau)$.

Proof. Let $\tau=\left\{U \in H S(X): U \in \mathcal{B}\left(x_{\lambda}\right)\right.$, for each $\left.x_{\lambda} \in U\right\}$. Then, clearly, $h^{\mathbf{0}} \in \tau$. By (HFN2), $h^{\mathbf{1}} \in \tau$. Thus, $\tau$ satisfies Axiom (HFT1).

Let $U, V \in \tau$, and let $x_{\lambda} \in U \widetilde{\cap} V$. Then, $x_{\lambda} \in U$ and $x_{\lambda} \in V$. Thus, $U, V \in \mathcal{B}\left(x_{\lambda}\right)$. By (HFN3), $U \widetilde{\cap} V \in \mathcal{B}\left(x_{\lambda}\right)$. Therefore, $U \widetilde{\cap} V \in \tau$. Hence, $\tau$ satisfies Axiom (HFT2).

Let $\left(U_{j}\right)_{j \in J} \subset \tau$, and let $x_{\lambda} \in \widetilde{U}_{j \in J} U_{j}$. Then, there is $j \in J$ such that $x_{\lambda} \in U_{j}$. Since $\left(U_{j}\right)_{j \in J} \subset \tau$, $U_{j} \in \mathcal{B}\left(x_{\lambda}\right)$. Thus, by (HFN2), $\widetilde{U}_{j \in J} U_{j} \in \mathcal{B}\left(x_{\lambda}\right)$. Therefore, $\widetilde{U}_{j \in J} U_{j} \in \tau$. Hence, $\tau$ satisfies Axiom (HFT3). Therefore, $\tau \in \operatorname{HFT}(X)$.

Now, we will show that $\mathcal{B}\left(x_{\lambda}\right)=H F N_{\tau}\left(x_{\lambda}\right)$. Let $N \in H F N_{\tau}\left(x_{\lambda}\right)$. Then, there is $U \in \tau$ such that $x_{\lambda} \in U \subset N$. Thus, by the definition of $\tau, U \in \mathcal{B}\left(x_{\lambda}\right)$, for each $\lambda \in U$. Therefore, by (HFN2), $N \in \mathcal{B}\left(x_{\lambda}\right)$. Hence, $\operatorname{HFN}_{\tau}\left(x_{\lambda}\right) \subset \mathcal{B}\left(x_{\lambda}\right)$. Suppose $N \in \mathcal{B}\left(x_{\lambda}\right)$, and let $U=\tilde{U}\left\{y_{\mu} \in H_{P}(X): N \in\right.$ $\left.\operatorname{HFN}_{\tau}\left(y_{\mu}\right)\right\}$. Then, clearly, $U \in H S(X)$ and $x_{\lambda} \in U$. By (HFN1), we can easily see that $U \subset N$. Let us show that $U \in \tau$, i.e., $U \in \mathcal{B}\left(y_{\mu}\right)$, for each $y_{\mu} \in U$. Let $y_{\mu} \in U$. By the definition of $U, N \in H F N_{\tau}\left(y_{\mu}\right)$. Since $H F N_{\tau}\left(y_{\mu}\right) \subset \mathcal{B}\left(y_{\mu}\right), N \in \mathcal{B}\left(y_{\mu}\right)$. Then, by (HFN4), there is $M \in \mathcal{B}\left(y_{\mu}\right)$ such that $N \in \mathcal{B}\left(z_{\nu}\right)$, for each $z_{v} \in M$. Thus, by the definition of $U, z_{v} \in U$. Therefore, $M \subset U$. Hence, by (HFN2), $U \in \mathcal{B}\left(y_{\mu}\right)$. This completes the proof.

Definition 14. Let $X$ be a nonempty set; let $x_{\lambda} \in H_{P}(X)$; and let $h \in H S(X)$. Then, $x_{\lambda}$ is said to:

(i) be quasi-coincident with $h$, denoted by $x_{\lambda} g h$, if $\lambda \supseteqq h^{c}(x)$,

(ii) be not quasi-coincident with $h$, denoted by $x_{\lambda} \bar{q} h$, if $\lambda \subset h^{c}(x)$.

Definition 15. Let $X$ be a nonempty set, and let $h_{1}, h_{2} \in H S(X)$. Then, $h_{1}$ is said to be quasi-coincident with $h_{2}$, denoted by $h_{1} q h_{2}$, if there is $x \in X$ such that $h_{1}(x) \supsetneqq h_{2}^{c}(x)$.

In this case, we say that $h_{1}$ and $h_{2}$ are quasi-coincident (with each other) at $x_{\lambda}$.

It is obvious that if $h_{1}$ and $h_{2}$ are quasi-coincident at $x_{\lambda}$, then $\left(h_{1} \widetilde{\cap} h_{2}\right)(x) \neq \phi$.

Theorem 4. Let $X$ be a nonempty set; let $x_{\lambda} \in H_{P}(X)$; and let $h, h_{1}, h_{2} \in H S(X)$. Then, $h_{1} \subset h_{2}$ if and only if $h_{1} \bar{q} h_{2}^{c}$. In particular, $x_{\lambda} \in h$ if and only if $x_{\lambda} \bar{q} h^{c}$. 
Proof. The proof follows from the fact: for each $x \in X$,

$$
h_{1} \subset h_{2} \text { iff } h_{1}(x) \supsetneqq h_{2}(x) \text { iff } h_{1}(x) \cup h_{2}(x)^{c} \varsubsetneqq h_{2}(x) \cup h_{2}(x)^{c}=\left(h_{2} \widetilde{\cup} h_{2}^{c}\right)(x)=[0,1] .
$$

Theorem 5. Let $X$ be a nonempty set; let $x_{\lambda} \in H_{P}(X)$; and let $\left(h_{j}\right)_{j \in J} \subset H S(X)$. Then, $x_{\lambda} q \widetilde{\cup}_{j \in J} h_{j}$ if and only if there is $j \in J$ such that $x_{\lambda} q h_{j}$.

Proof. Suppose $x_{\lambda} q \widetilde{\cup}_{j \in J} h_{j}$. Then, $\lambda \supseteqq\left(\widetilde{\cup}_{j \in J} h_{j}\right)^{c}(x)=\left(\widetilde{\cap}_{j \in J} h_{j}^{c}\right)(x)=\bigcap_{j \in J} h_{j}(x)^{c}$. Thus, there is $j \in J$ such that $\lambda \supseteqq h_{j}(x)^{c}$.

Conversely, suppose there is $j \in J$ such that $\lambda \supseteqq h_{j}(x)^{c}$. Then, $\lambda \supseteqq \bigcap_{j \in J} h_{j}(x)^{c}$ and $\bigcap_{j \in J} h_{j}(x)^{c}=$ $\left(\widetilde{U}_{j \in J} h_{j}\right)^{c}(x)$. Thus, $x_{\lambda} q \widetilde{U}_{j \in J} h_{j}$.

Proposition 8. Let $X, Y$ be nonempty sets; let $x_{\lambda} \in H_{P}(X)$; let $h \in H S(X)$; and let $f: X \rightarrow Y$ be a mapping. If $x_{\lambda} q h$, then $f\left(x_{\lambda}\right) q f(h)$.

Proof. Suppose $x_{\lambda} g h$. Then, $\lambda \supseteqq h^{c}(x)=h(x)^{c}$ and $f(h)(f(x))=h(x)$. Thus,

$$
f(h)^{c}(f(x))=f(h)(f(x))^{c}=h(x)^{c} \varsubsetneqq \lambda=f(x)_{\lambda}(f(x))=f(x)_{\lambda}(f(x)) .
$$

Therefore, $f(x)_{\lambda} q f(h)$.

Definition 16. Let $(X, \tau)$ be a hesitant fuzzy topological space; let $N \in H S(X)$; and let $x_{\lambda} \in H_{P}(X)$. Then, $N$ is called an $H Q$-neighborhood (HQN) of $x_{\lambda}$, if there is a $U \in \tau$ such that $x_{\lambda} q U \subset N$. The set of all $H Q$-neighborhoods of $x_{\lambda}$ is called the system of of HQ-neighborhoods of $x_{\lambda}$ and denoted by $H Q N\left(x_{\lambda}\right)$.

Proposition 9. Let $(X, \tau)$ be a hesitant fuzzy topological space, and let $x_{\lambda} \in H_{P}(X)$. Then, $H Q N\left(x_{\lambda}\right)$ has the following properties:

(HQN1) $x_{\lambda} q N$, for each $N \in H Q N\left(x_{\lambda}\right)$,

(HQN2) if $N \in H Q N\left(x_{\lambda}\right)$, then $M \in H Q N\left(x_{\lambda}\right)$, for each $M \in H S(X)$ such that $N \subset M$,

(HQN3) if $N, M \in H Q N\left(x_{\lambda}\right)$, then $N \widetilde{\cap} M \in H Q N\left(x_{\lambda}\right)$,

(HQN4) if $N \in H Q N\left(x_{\lambda}\right)$, then there is $M \in H Q N\left(x_{\lambda}\right)$ such that $N \in H Q N\left(y_{\mu}\right)$, for each $y_{\mu} q M$.

Proof. The proofs are similar to Proposition 6.

Proposition 10. Let $X$ be a nonempty set, and let for each $x_{\lambda} \in H_{P}(X)$ there be a nonempty collection $\mathcal{B}\left(x_{\lambda}\right)$ of hesitant fuzzy sets in X satisfying Properties (HQN1)-(HQN4). Then, there is a unique HFT $\tau$ on $X$ such that for each $x_{\lambda} \in H_{P}(X), \mathcal{B}\left(x_{\lambda}\right)=H Q N_{\tau}\left(x_{\lambda}\right)$, where $H Q N_{\tau}\left(x_{\lambda}\right)$ denotes the set of all HQNs of $x_{\lambda}$ in $(X . \tau)$.

Proof. The proof is similar to Proposition 7.

Theorem 6. Let $(X, \tau)$ be a hesitant fuzzy topological space, and let $\mathcal{B} \subset H S(X)$. Then, $\mathcal{B}$ is a base for $\tau$ if and only if for each $x_{\lambda} \in H_{P}(X)$ and each $U \in H Q N\left(x_{\lambda}\right)$ such that $U \in \tau$, there is $B \in \mathcal{B}$ such that $x_{\lambda} q B \subset U$.

Proof. The necessary condition follows directly from the definition of a base and Proposition 8 . Let us show that the sufficient condition. Assume that $\mathcal{B}$ is not a base for $\tau$. Then, there is $A \in \mathcal{B}$ such that $V=\widetilde{\cup}\{B \in \mathcal{B}: B \subset A\} \neq A$. Thus, there is $x \in X$ such that $V(x) \varsubsetneqq A(x)$. Let $\lambda=[0,1] \backslash V(x)=V^{c}(x)$. Then, clearly, $A(x) \cup \lambda \supsetneqq V(x) \cup \lambda=[0,1]$. Thus, $A(x) \cup \lambda \supsetneqq V(x)=[0,1]$. Therefore, $x_{\lambda} q_{A}$. 
On the other hand, for any $B \in \mathcal{B}$ such that $B \subset A, A \subset V$. Then, $B(x) \cup \lambda \subset V(x) \cup \lambda=[0,1]$. Thus, $x_{\lambda} \bar{q} B$. Since $B \subset A, x_{\lambda} \bar{q} A$. Therefore, this contradicts the assumption. This completes the proof.

Definition 17. Let $(X, \tau)$ be a hesitant fuzzy topological space, and let $h \in H S(G)$. Then, the hesitant fuzzy interior points of $h$, denoted by int $t_{H}(h)$, are a hesitant fuzzy set in $X$ defined by:

$$
\operatorname{int}_{H}(h)=\widetilde{\bigcup}\{U \in \tau: U \subset h\} .
$$

Example 5. Let $X=\{a, b, c\}$, and let $\tau=\left\{h^{\mathbf{0}}, h^{\mathbf{1}}, h_{1}, h_{2}, h_{3}, h_{4}\right\} \in \operatorname{HFT}(X)$, where $h_{1}(a)=\{0.4\} \cup[0.5,1), h_{1}(b)=(0.1,0.9], h_{1}(c)=[0.3,0.8) \cup\{0.1,0.9\}$, $h_{2}(a)=(0.3,0.5) \cup[0.6,0.9], h_{2}(b)=\{0.2\} \cup(0.8,0.9), h_{2}(c)=(0.4,1] \cup\{0.1\}$, $h_{3}(a)=(0.3,1), h_{3}(b)=(0.1,0.9], h_{3}(c)=[0.3,1] \cup\{0.1\}$, $h_{4}(a)=\{0.4\} \cup[0.6,0.9], h_{3}(b)=\{0.2\} \cup(0.8,0.9), h_{3}(c)=(0.4,0.8) \cup\{0.1,0.9\}$.

Let $h$ be the hesitant fuzzy set in $X$ given by:

$$
h(a)=[0.4,1), h(b)=(0.1,0.9], h(c)=[0.1,0.8) \cup\{0.9\} .
$$

Then, clearly $h^{0} \subset h, h_{1} \subset h$ and $h_{4} \subset h$. Thus, int $H_{H}(h)=h^{\mathbf{0}} \widetilde{\cup} h_{1} \widetilde{\cup} h_{4}=h_{1} \widetilde{\cup} h_{4}$.

From Definition 17, we have the following result.

Proposition 11. Let $(X, \tau)$ be a hesitant fuzzy topological space, and let $h, h_{1} h_{2} \in H S(X)$. Then,

(1) $\operatorname{int}_{H}\left(h^{0}\right)=h^{\mathbf{0}}$,

(2) int $_{H}(h)$ is the largest hesitant fuzzy open set contained in $h$,

(3) $h \in \tau$ if and only if int ${ }_{H}(h)=h$,

(4) if $h_{1} \subset h_{2}$, then int ${ }_{H}\left(h_{1}\right) \subset \operatorname{int}_{H}\left(h_{2}\right)$.

Theorem 7. Let $(X, \tau)$ be a hesitant fuzzy topological space; let $x_{\lambda} \in H_{P}(X)$; and let $A \in H S(X)$. Then, $x_{\lambda} \in \operatorname{int}_{H}(A)$ if and only if there is $N \in \operatorname{HFN}\left(x_{\lambda}\right)$ such that $N \subset A$.

Proof. The proof is straightforward.

Proposition 12. (Hesitant fuzzy interior axioms). Let $(X, \tau)$ be a hesitant fuzzy topological space, and let $h, h_{1} h_{2} \in H S(X)$. Then:

(HFI1) $\operatorname{int}_{H}\left(h^{\mathbf{1}}\right)=h^{\mathbf{1}}$,

(HFI2) $\operatorname{int}_{H}(h) \subset h$,

(HFI3) $\operatorname{int}_{H}\left(\operatorname{int}_{H}(h)\right)=\operatorname{int}_{H}(h)$,

(HFI4) $i n t_{H}\left(h_{1} \widetilde{\cap} h_{2}\right)=i n t_{H}\left(h_{1}\right) \widetilde{n} i n t_{H}\left(h_{2}\right)$.

Proof. From Definition 17 and Proposition 11 (1), the proofs of (HFI1) and (HFI2) are clear.

(HFI3) By (HFI2), it is obvious that int ${ }_{H}\left(\right.$ int $\left._{H}(h)\right) \subset \operatorname{int}_{H}(h)$. Let $x_{\lambda} \in$ int $_{H}(h)$. Then, there is $U \in \tau$ such that $x_{\lambda} \in U \subset h$. Thus, by Proposition 11 (2) and (3) and (HFI2), $x_{\lambda} \in U \subset$ int $_{H}(h)$. Therefore, $x_{\lambda} \in \operatorname{int}_{H}\left(\operatorname{int}_{H}(h)\right)$. Hence, int ${ }_{H}(h) \subset \operatorname{int}_{H}\left(\right.$ int $\left._{H}(h)\right)$. Therefore, int ${ }_{H}\left(\right.$ int $\left._{H}(h)\right)=$ int $_{H}(h)$.

(HFI4) It is clear that $h_{1} \widetilde{\cap} h_{2} \subset h_{1}$ and $h_{1} \widetilde{\cap} h_{2} \subset h_{2}$. Then, by Proposition 11 (3), int $t_{H}\left(h_{1} \widetilde{\cap} h_{2}\right) \subset$ $\operatorname{int}_{H}\left(h_{1}\right)$ and $\operatorname{int}_{H}\left(h_{1} \widetilde{\cap} h_{2}\right) \subset \operatorname{int}_{H}\left(h_{2}\right)$. Thus, int ${ }_{H}\left(h_{1} \widetilde{\cap} h_{2}\right) \subset \operatorname{int}_{H}\left(h_{1}\right) \widetilde{\cap} i n t_{H}\left(h_{2}\right)$. Let $x_{\lambda} \in$ int $_{H}\left(h_{1}\right) \widetilde{n i n t}_{H}\left(h_{2}\right)$. Then, $x_{\lambda} \in \operatorname{int}_{H}\left(h_{1}\right)$ and $x_{\lambda} \in$ int $_{H}\left(h_{2}\right)$. Thus, there are $U, V \in \tau$ such that $x_{\lambda} \in$ $U \subset h_{1}$ and $x_{\lambda} \in V \subset h_{2}$. Therefore, $x_{\lambda} \in U \widetilde{\cap} V \subset h_{1} \widetilde{\cap} h_{2}$ and $U \widetilde{\cap} V \in \tau$. Hence, $x_{\lambda} \in \operatorname{int}_{H}\left(h_{1} \widetilde{\cap} h_{2}\right)$, i.e., $\operatorname{int}_{H}\left(h_{1}\right) \widetilde{\cap} i n t_{H}\left(h_{2}\right) \subset \operatorname{int}_{H}\left(h_{1} \widetilde{\cap} h_{2}\right)$. Therefore, $i n t_{H}\left(h_{1} \widetilde{\cap} h_{2}\right)=i n t_{H}\left(h_{1}\right) \widetilde{n} i n t_{H}\left(h_{2}\right)$.

Definition 18. Let $X$ be a nonempty set. Then, a mapping ${ }^{\circ} H: H S(X) \rightarrow H S(X)$ is called a hesitant fuzzy interior operator on $X$, if it satisfies Properties (HFI1)-(HFI4) of Proposition 12. 
The following result shows that a hesitant fuzzy interior operator completely determines a hesitant fuzzy topology on $X$ and that in this topology, the operator is the hesitant fuzzy interior.

Proposition 13. Let ${ }^{\circ} \mathrm{H}$ be hesitant fuzzy interior operator on a set X. Let:

$$
\tau=\left\{U \in H S(X): U^{\circ}=U\right\} .
$$

Then, $\tau \in \operatorname{HFT}(X)$, and if int ${ }_{H}$ is the hesitant fuzzy interior defined by $\tau$, then

$$
U^{{ }^{\circ}}=\operatorname{int}_{H}(U) \text {, for each } U \in H S(X) .
$$

Proof. By (HFI1) and (HFI2), $\left(h^{\mathbf{1}}\right)^{\circ_{H}}=h^{\mathbf{1}}$ and $\left(h^{0}\right)^{\circ} H=h^{0}$. Then, $h^{0}, h^{\mathbf{1}} \in \tau$. Thus, $\tau$ satisfies Axiom (HFT1).

Let $U, V \in \tau$. Then, $U^{{ }^{H}}=U$ and $V^{{ }^{H}}=V$. Thus, by (HFI4),

$$
(U \widetilde{\cap} V)^{\circ} H=U^{\circ} H \widetilde{\cap} V^{\circ} H=U \widetilde{\cap} V .
$$

Therefore, $U \widetilde{\cap} V \in \tau$. Hence, $\tau$ satisfies Axiom (HFT2).

Let $\left(U_{j}\right)_{j \in J} \subset \tau$, and let $U=\widetilde{U}_{j \in J} U_{j}$. Then clearly, $U_{j} \subset U$, for each $j \in J$. By (HFI2), $U_{j}^{\circ H} \subset U^{\circ H}$, for each $j \in J$. Thus, $\widetilde{U}_{j \in J} U_{j}^{\circ H} \subset U^{{ }^{\circ} H}$. Since $\left(U_{j}\right)_{j \in J} \subset \tau, U_{j}^{\circ H}=U_{j}$, for each $j \in J$. Therefore, $U \subset U^{\circ} H$, i.e., $U^{\circ} H=U$. Hence, $\tau$ satisfies Axiom (HFT3). Therefore, $\tau \in H F T(X)$.

Suppose $i n t_{H}$ is the hesitant fuzzy interior defined by $\tau$, and let $h \in H S(X)$. Then clearly, $\operatorname{int}_{H}(h)$ is the largest hesitant fuzzy open set in X contained in $h$. Thus, $h^{\circ H}=h$. By (HFI3), $h^{\circ} H\left(h^{\circ} H\right)=$ $h^{\circ}$. Therefore, $h^{\circ} \in \tau$. By (HFI2), $h^{\circ H} \subset h$. Hence, $h^{\circ} \subset$ int $_{H}(h)$. On the other hand, by $\left(\right.$ HFI2), int $t_{H}(h) \subset h$. Then, $h^{\circ} C \subset h^{\circ} H\left(\operatorname{int}_{H}(h)\right)=\operatorname{int}_{H}(h)$. Thus, $h^{\circ} H=$ int $_{H}(h)$. Therefore, $U^{\circ} H=$ $\operatorname{int}_{H}(U)$, for each $U \in H S(X)$.

Definition 19. Let $(X, \tau)$ be a hesitant fuzzy topological space, and let $h \in H S(X)$. Then, the hesitant fuzzy closure of $h$, denoted by $\mathrm{cl}_{H}(h)$, is a hesitant fuzzy set in $X$ defined by:

$$
c l_{H}(h)=\widetilde{\bigcap}\{F \in H F C(X): h \subset F\} .
$$

Example 6. Let $X=\{a, b, c\}$, and let $\tau=\left\{h^{0}, h^{\mathbf{1}}, h_{1}, h_{2}, h_{3}, h_{4}\right\} \in \operatorname{HFT}(X)$, where $h_{1}(a)=\{0.4\} \cup[0.5,1), h_{1}(b)=(0.1,0.9], h_{1}(c)=[0.3,0.8) \cup\{0.1,0.9\}$,

$h_{2}(a)=(0.3,0.5) \cup[0.6,0.9], h_{2}(b)=\{0.2\} \cup(0.8,0.9), h_{2}(c)=(0.4,1] \cup\{0.1\}$,

$h_{3}(a)=(0.3,0.5) \cup[0.5,1), h_{3}(b)=(0.1,0.9], h_{3}(c)=[0.3,1] \cup\{0.1\}$,

$h_{4}(a)=\{0.4\} \cup[0.6,0.9], h_{3}(b)=\{0.2\} \cup(0.8,0.9), h_{3}(c)=(0.4,0.8) \cup\{0.1,0.9\}$.

Then, $\operatorname{HFC}(X)=\left\{h^{0}, h^{\mathbf{1}}, h_{1}^{c}, h_{2}^{c}, h_{3}^{c}, h_{4}^{c}\right\}$,

where $h_{1}^{c}(a)=[0,0.4) \cup(0.4,0.5) \cup\{1\}, h_{1}^{c}(b)=[0,0.1) \cup[0.9,1]$,

$h_{1}^{c}(c)=[0,0.1) \cup(0.1,0.3) \cup[0.8,0.9) \cup(0.9,1]$,

$h_{2}^{c}(a)=[0,0.3] \cup[0.5,0.6) \cup(0.9,1], h_{2}^{c}(b)=[0,0.2) \cup(0.2,0.8] \cup[0.9,1]$,

$h_{2}^{c}(c)=[0,0.1] \cup(0.1,0.4]$,

$h_{3}^{c}(a)=[0,0.3] \cup\{1\}, h_{3}^{c}(b)=[0,0.1] \cup(0.9,1], h_{3}^{c}(c)=[0,0.1) \cup(0.1,0.3)$,

$h_{4}^{c}(a)=[0,0.4) \cup(0.4,0.6) \cup(0.9,1], h_{4}^{c}(b)=[0,0.2) \cup(0.2,0.8] \cup[0.9,1]$,

$h_{4}^{c}(c)=[0,0.1) \cup(0.1,0.4] \cup[0.8,0.9) \cup(0.9,1]$.

Let $h$ be the hesitant fuzzy set in $X$ given by:

$$
h(a)=[0,0.2] \cup(0.9,1], h(b)=(0.9,1], h(c)=[0,0.2) \cup[0.9,1] .
$$

Then clearly, $h \subset h^{\mathbf{1}}, h \subset h_{1}^{c}$ and $h \subset h_{3}^{c}$. Thus, $c l_{H}(h)=h^{\mathbf{0}} \widetilde{\cap} h_{1}^{c} \widetilde{\cap} h_{3}^{c}=h_{1}^{c} \widetilde{n} h_{3}^{c}$.

From Definition 19, we have the following result. 
Proposition 14. Let $(X, \tau)$ be a hesitant fuzzy topological space, and let $h, h_{1} h_{2} \in H S(X)$. Then,

(1) $c l_{H}\left(h^{\mathbf{1}}\right)=h^{\mathbf{1}}$,

(2) $c l_{H}(h)$ is the smallest hesitant fuzzy closed set containing $h$,

(3) $h \in H F C(X)$ if and only if $c l_{H}(h)=h$,

(4) if $h_{1} \subset h_{2}$, then $c l_{H}\left(h_{1}\right) \subset c l_{H}\left(h_{2}\right)$.

Theorem 8. Let $(X, \tau)$ be a hesitant fuzzy topological space; let $x_{\lambda} \in H_{P}(X)$; and let $A \in H S(X)$. Then, $x_{\lambda} \in c_{H}(A)$ if and only if for each $N \in H Q N\left(x_{\lambda}\right), N q A$.

In this case, $x_{\lambda}$ is called a hesitant fuzzy closure point of $A$. In fact,

$$
c l_{H}(A)=\widetilde{\bigcup}\left\{x_{\lambda} \in H_{P}(X): x_{\lambda} \text { is a hesitant fuzzy closure point of } A\right\} .
$$

Proof. $x_{\lambda} \in c l_{H}(A)$ iff for each $F \in H F C(X)$ with $F \supset A, x_{\lambda} \in F$, i.e., $\lambda \subset F(x)$

iff for each $F^{c}=U \in \tau$ with $U \subset A^{c}, U(x) \subset A(x)^{c}$

iff for each $U \in \tau$ with $U(x) \supsetneqq A^{c}(x), U \not \subset A^{c}$

iff by Theorem $4, U q A$

iff for each $N \in H Q N\left(x_{\lambda}\right), N q A$.

Proposition 15. Let $(X, \tau)$ be a hesitant fuzzy topological space, and let $h \in H S(X)$. Then:

$$
\operatorname{int}_{H}(h)=\left(c l_{H}\left(h^{c}\right)\right)^{c} \text {, i.e., }\left(\operatorname{int}_{H}(h)\right)^{c}=c l_{H}\left(h^{c}\right) .
$$

Proof. Let $x_{\lambda} \in \operatorname{int}_{H}(h)$. Then clearly, $x_{\lambda} \in \operatorname{int}_{H}(h) \in \tau$ such that $\operatorname{int}_{H}(h) \widetilde{\cap} h^{c}=h^{0}$. Thus, $\lambda \supsetneqq h^{c}(x)$, i.e., $x_{\lambda} \notin h^{c}$. Since $h^{c} \subset c l_{H}\left(h^{c}\right), x_{\lambda} \notin c l_{H}\left(h^{c}\right)$. Therefore, $\lambda \in\left(c l_{H}\left(h^{c}\right)\right)^{c}$. Hence, int $t_{H}(h) \subset\left(c l_{H}\left(h^{c}\right)\right)^{c}$.

Now, let $x_{\lambda} \in\left(c l_{H}\left(h^{c}\right)\right)^{c}$. Then, $x_{\lambda} \notin c l_{H}\left(h^{c}\right)$. Thus, $x_{\lambda} \notin h^{c}$, i.e., $x_{\lambda} \in h$. Since $x_{\lambda} \notin c l_{H}\left(h^{c}\right)$, there is $U \in \tau$ such that $x_{\lambda} \in U$ and $U \widetilde{\cap} h^{c}=h^{0}$. Therefore, $U \subset h$. Hence, $x_{\lambda} \in \operatorname{int}_{H}(h)$, i.e., $\left(c l_{H}\left(h^{c}\right)\right)^{c} \subset \operatorname{int}_{H}(h)$. Therefore, $\operatorname{int}_{H}(h)=\left(c l_{H}\left(h^{c}\right)\right)^{c}$.

From Definition 19 and Proposition 15, we have the following result.

Proposition 16. (Hesitant fuzzy Kuratowski closure axioms). Let $(X, \tau)$ be a hesitant fuzzy topological space, and let $h, h_{1} h_{2} \in H S(X)$. Then:

(HFC1) $c l_{H}\left(h^{0}\right)=h^{0}$,

(HFC2) $h \subset c l_{H}(h)$,

(HFC3) $c l_{H}\left(c l_{H}(h)\right)=c l_{H}(h)$,

$(\mathrm{HFC} 4) c l_{H}\left(h_{1} \widetilde{\cup} h_{2}\right)=c l_{H}\left(h_{1}\right) \widetilde{\cup} c l_{H}\left(h_{2}\right)$.

Definition 20. Let $X$ be a nonempty set. Then, a mapping ${ }^{-H}: H S(X) \rightarrow H S(X)$ is called a hesitant fuzzy closure operator on X, if it satisfies Properties (HFC1)-(HFC4) of Proposition 16.

As expected, a result analogous to Proposition 13 holds for the hesitant fuzzy closure operator. Then, a hesitant fuzzy closure operator completely determines a hesitant fuzzy topology, and in that topology, the hesitant fuzzy closure operator is the hesitant fuzzy closure.

Proposition 17. Let ${ }^{-H}$ be a hesitant fuzzy closure operator on a set $X$. Let $\mathcal{F}=\left\{F \in H S(X): F^{-H}=F\right\}$, and let $\tau=\left\{U \in H S(X): U^{c} \in \mathcal{F}\right\}$. Then, $\tau \in H F T(X)$, and if $c l_{H}$ is the hesitant fuzzy closure defined by $\tau$, then:

$$
F^{-H}=c l_{H}(F) \text {, for each } F \in H S(X) .
$$

Proof. It is similar to Proposition 13. 
Definition 21. Let $X$ be a nonempty set, and let $h_{1}, h_{2} \in H S(X)$. Then, the difference of $h_{1}$ and $h_{2}$, denoted by $h_{1} \backslash h_{2}$, is a hesitant fuzzy set in $X$ defined by:

$$
h_{1} \backslash h_{2}=h_{1} \widetilde{\cap} h_{2}^{c}
$$

Definition 22. Let $(X, \tau)$ be a hesitant fuzzy topological space, and let $h \in H S(X)$. Then, $x_{\lambda} \in H_{P}(X)$ is called a hesitant fuzzy limit point or an accumulation point of $h$, if $h \widetilde{\cap} U \backslash\left\{x_{\lambda}\right\} \neq h^{0}$, for each $U \in \tau$ with $x_{\lambda} \in U$, where $U \backslash\left\{x_{\lambda}\right\}=U \widetilde{\cap}\left\{x_{\lambda}\right\}^{c}$. The union of all hesitant fuzzy limit points of $h$ will be called the hesitant fuzzy derived set of $h$ and will be denoted by $D_{H}(h)$. Then clearly,

$$
D_{H}(h)=\widetilde{\bigcup}\left\{x_{\lambda} \in H_{P}(X): x_{\lambda} \text { is a hesitant fuzzy limit point of } h\right\} .
$$

Example 7. (1) Let $X$ be the hesitant fuzzy discrete space. Then, $D_{H}(h)=h^{0}$, for each $h \in H S(X)$.

(2) Let $X$ be the hesitant fuzzy indiscrete space. Then, $D_{H}(h)=h^{1}$, for each $h \in H S(X)$.

From Definition 22, we have the following result.

Proposition 18. Let $(X, \tau)$ be a hesitant fuzzy topological space, and let $h, h_{1}, h_{2} \in H S(X)$. Then:

(HFD1) $D_{H}\left(h^{0}\right)=h^{0}$,

(HFD2) if $h_{1} \subset h_{2}$, then $D_{H}\left(h_{1}\right) \subset D_{H}\left(h_{2}\right)$,

(HFD3) if $x_{\lambda} \in D_{H}(h)$, then $x_{\lambda} \in D_{H}\left(h \backslash\left\{x_{\lambda}\right\}\right)$,

(HFD4) $D_{H}\left(h_{1} \widetilde{\cup} h_{2}\right)=D_{H}\left(h_{1}\right) \widetilde{\cup} D_{H}\left(h_{2}\right)$.

Definition 23. Let $(X, \tau)$ be a hesitant fuzzy topological space, and let $h \in H S(X)$. Then, the hesitant fuzzy exterior of $h$, denoted by $e_{H}(h)$, is a hesitant fuzzy set in $X$ defined by $e_{H}(h)=\operatorname{int}_{H}\left(h^{c}\right)$.

It is obvious that $\operatorname{int}_{H}(h)=e_{H}\left(h^{c}\right)$.

By the above definition, we have the following result.

Proposition 19. Let $(X, \tau)$ be a hesitant fuzzy topological space, and let $h, h_{1}, h_{2} \in H S(X)$. Then:

(HFD1) $e_{H}\left(h^{0}\right)=h^{1}$,

(HFD2) $e_{H}(h) \subset h^{c}$,

(HFD3) $e_{H}(h)=e_{H}\left(\left[e_{H}(h)\right]^{c}\right)$,

(HFD4) $e_{H}\left(h_{1} \widetilde{\cup} h_{2}\right)=e_{H}\left(h_{1}\right) \widetilde{\cap} e_{H}\left(h_{2}\right)$.

Definition 24. Let $(X, \tau)$ be a hesitant fuzzy topological space, and let $h \in H S(X)$. Then, the hesitant fuzzy boundary of $h$, denoted by $b_{H}(h)$, is a hesitant fuzzy set in X defined by $b_{H}(h)=\left[\operatorname{int}_{H}(h)\right]^{c} \widetilde{\cup} \operatorname{int}_{H}\left(h^{c}\right)$.

It is clear that $b_{H}(h)=b_{H}\left(h^{c}\right)=c l_{H}(h) \widetilde{\cap} c l_{H}\left(h^{c}\right)=c l_{H}(h) \backslash i n t_{H}(h)$.

\section{Hesitant Fuzzy Continuous Mappings}

We define a hesitant fuzzy continuous mapping and a hesitant fuzzy open (resp. closed) mapping and prove that each concept has similar properties in classical topological spaces.

Definition 25. Let $(X, \tau),(Y, \sigma)$ be hesitant fuzzy topological spaces. Then, a mapping $f:(X, \tau) \rightarrow(Y, \sigma)$ is said to be hesitant fuzzy continuous, if $f^{-1}(V) \in \tau$, for each $V \in \sigma$.

From Result 2 and Definition 25, we have the following result.

Proposition 20. Let $(X, \tau),(Y, \sigma),(Z, \delta)$ be hesitant fuzzy topological spaces.

(1) The identity mapping id $:(X, \tau) \rightarrow(X, \tau)$ is continuous. 
(2) If $f:(X, \tau) \rightarrow(Y, \sigma)$ and $g:(Y, \sigma) \rightarrow(Z, \delta)$ are continuous, then $g \circ f:(X, \tau) \rightarrow(Z, \delta)$ is continuous.

Remark 2. From Proposition 20, we can see that the class of all hesitant fuzzy topological spaces and hesitant fuzzy continuous mappings forms a concrete category.

Definition 26. Let $(X, \tau),(Y, \sigma)$ be hesitant fuzzy topological spaces. Then, a mapping $f:(X, \tau) \rightarrow(Y, \sigma)$ is said to be hesitant fuzzy continuous at $x_{\lambda} \in H_{P}(X)$, if $f^{-1}(V) \in \operatorname{HFN}\left(x_{\lambda}\right)$, for each $V \in \operatorname{HFN}\left(f\left(x_{\lambda}\right)\right)=$ $\operatorname{HFN}\left(f(x)_{\lambda}\right)$ (see Remark 1).

The following is the immediate result of Result 2 and Definitions 25 and 26.

Theorem 9. Let $(X, \tau),(Y, \sigma)$ be hesitant fuzzy topological spaces. Then, a mapping $f:(X, \tau) \rightarrow(Y, \sigma)$ is hesitant fuzzy continuous if and only if $f$ is hesitant fuzzy continuous at each hesitant fuzzy point of $X$.

Theorem 10. Let $(X, \tau),(Y, \sigma)$ be hesitant fuzzy topological spaces, and let $f:(X, \tau) \rightarrow(Y, \sigma)$ be a mapping. Then, the following are equivalent:

(1) $f$ is continuous,

(2) $f^{-1}(F) \in \operatorname{HFC}(X)$, for each $F \in \operatorname{HFC}(Y)$,

(3) $f^{-1}(S) \in \tau$, for each $S \in \mathcal{S}$, where $\mathcal{S}$ is the sub-base for $\sigma$,

(4) $f$ is continuous at each $x_{\lambda} \in H_{P}(X)$,

(5) for each $x_{\lambda} \in H_{P}(X)$ and each $V \in \operatorname{HFN}\left(f(x)_{\lambda}\right)$, there is $U \in H F N\left(x_{\lambda}\right)$ such that $f(U) \subset V$,

(6) $f\left(c l_{H}(h)\right) \subset c l_{H}(f(h))$, for each $h \in H S(X)$,

(7) $c l_{H}\left(f^{-1}(h)\right) \subset f^{-1}\left(c l_{H}(h)\right)$, for each $h \in H S(Y)$.

Proof. $(1) \Rightarrow(2)$ : The proof is clear from Definitions 8 and 25.

$(2) \Rightarrow(3)$ : Suppose $f^{-1}(F) \in \operatorname{HFC}(X)$, for each $F \in \operatorname{HFC}(Y)$, and let $S \in \mathcal{S}$. Then, clearly, $S^{c} \in \operatorname{HFC}(Y)$. Thus, by the hypothesis, $f^{-1}\left(S^{c}\right) \in \operatorname{HFC}(X)$. It is obvious that $f^{-1}\left(S^{c}\right)=\left(f^{-1}(S)\right)^{c}$. Therefore, $\left(f^{-1}(S)\right)^{c} \in \operatorname{HFC}(X)$. Hence, $f^{-1}(S) \in \tau$.

(3) $\Rightarrow$ (4): Suppose (3); and let $x_{\lambda} \in H_{P}(X)$, and let $V \in \operatorname{HFN}\left(f(x)_{\lambda}\right)$. Then, there is $S \in \mathcal{S}$ such that $f(x)_{\lambda} \in S \subset V$, where $\mathcal{S}$ is the sub-base for $\sigma$. Thus, by (3), $f^{-1}(S) \in \tau$. Since $f(x)_{\lambda} \in S \subset V$, $x_{\lambda} \in f^{-1}(S) \subset f^{-1}(V)$. Therefore, $f^{-1}(V) \in \operatorname{HFN}\left(x_{\lambda}\right)$. Hence, $f$ is continuous at each $x_{\lambda} \in H_{P}(X)$.

$(4) \Rightarrow(5)$ : The proof is obvious.

$(5) \Rightarrow$ (6): Suppose (5), and let $y_{\mu} \in f\left(c l_{H}(h)\right)$, for each $h \in H S(X)$. Then, there is $x \in X$ such that $y_{\mu}=f\left(x_{\mu}\right)$ and $x_{\mu} \in c_{H}(h)$. Let $V \in \operatorname{HFN}\left(f\left(x_{\mu}\right)\right)$. Then, by (5), there is $U \in \operatorname{HFN}\left(x_{\mu}\right)$ such that $f(U) \subset V$. Since $x_{\mu} \in c l_{H}(h), U \widetilde{\cap} h \neq h^{\mathbf{0}}$. Thus, $f(U) \widetilde{\cap} f(h) \neq h^{\mathbf{0}}$. Therefore, $V \widetilde{\cap} f(h) \neq h^{\mathbf{0}}$. Hence, $y_{\mu} \in c l_{H}(f(h))$. Therefore, $f\left(c l_{H}(h)\right) \subset c l_{H}(f(h))$.

(6) $\Rightarrow$ (7): Suppose (6), and let $A=f^{-1}(h)$, for each $h \in H S(Y)$. Then, by (6), $f\left(c l_{H}(A)\right) \subset$ $c l_{H}(f(A))$. Thus, $f\left(c l_{H}\left(f^{-1}(h)\right)\right) \subset c l_{H}\left(f\left(f^{-1}(h)\right) \subset c l_{H}(h)\right.$. Therefore, $c l_{H}\left(f^{-1}(h)\right) \subset f^{-1}\left(c l_{H}(h)\right)$.

$(7) \Rightarrow(2)$ : The proof is clear.

The following is the immediate result of Theorem 10.

Corollary 1. $f:(X, \tau) \rightarrow(Y, \sigma)$ is continuous if and only if $f^{-1}\left(\right.$ int $\left._{H}(h)\right) \subset$ int $_{H}\left(f^{-1}(h)\right)$, for each $h \in H S(Y)$.

Definition 27. Let $(X, \tau),(Y, \sigma)$ be hesitant fuzzy topological spaces. Then, a mapping $f:(X, \tau) \rightarrow(Y, \sigma)$ is said to be hesitant fuzzy open (resp. closed), if $f(U) \in \sigma$, for each $U \in \tau$ (resp. $f(F) \in H F C(Y)$, for each $F \in \operatorname{HFC}(X))$.

Proposition 21. Let $(X, \tau),(Y, \sigma),(Z, \delta)$ be hesitant fuzzy topological spaces. If $f:(X, \tau) \rightarrow(Y, \sigma)$ and $g:(Y, \sigma) \rightarrow(Z, \delta)$ are open (resp. closed), then $g \circ f:(X, \tau) \rightarrow(Z, \delta)$ is open (resp. closed). 
Proof. Let $U \in \tau$. Since $f:(X, \tau) \rightarrow(Y, \sigma)$ is open, $f(U) \in \sigma$. Since $g:(Y, \sigma) \rightarrow(Z, \delta)$ is open, $g(f(U)) \in \delta$. Then, $(g \circ f)(U) \in \delta$. Thus, $g \circ f$ is open. The proof of the second part is similar.

Theorem 11. Let $(X, \tau),(Y, \sigma)$ be hesitant fuzzy topological spaces, and let $f: X \rightarrow Y$ be a mapping. Then, $f$ is open if and only if $f\left(\operatorname{int}_{H}(h)\right) \subset \operatorname{int}_{H}(f(h))$, for each $h \in H S(X)$.

Proof. Suppose $f$ is open, and let $h \in H S(X)$. Then, clearly, int $t_{H}(h) \in \tau$. Thus, by the hypothesis, $f\left(\operatorname{int}_{H}(h)\right) \in \sigma$. Since $\operatorname{int}_{H}(h) \subset h, f\left(\operatorname{int}_{H}(h) \subset f(h)\right.$. Since $\operatorname{int}_{H}(f(h))$ is the largest hesitant fuzzy open set contained in $f(h), f\left(\operatorname{int}_{H}(h) \subset \operatorname{int}_{H}(f(h))\right.$.

Conversely, assume the necessary condition holds, and let $U \in \tau$. Then, clearly, $U=\operatorname{int}_{H}(U)$. Thus, by the hypothesis, $f(U)=f\left(\operatorname{int}_{H}(U)\right) \subset \operatorname{int}_{H}(f(U))$. Since $\operatorname{int}_{H}(f(U)) \subset f(U), f(U)=$ $\operatorname{int}_{H}(f(U))$. Therefore, $f(U) \in \sigma$. Hence, $f$ is open.

Proposition 22. Let $(X, \tau),(Y, \sigma)$ be hesitant fuzzy topological spaces, and let $f: X \rightarrow Y$ be an injective mapping. If $f:(X, \tau) \rightarrow(Y, \sigma)$ is continuous, then $\operatorname{int}_{H}(f(h)) \subset f\left(\right.$ int $\left._{H}(h)\right)$, for each $h \in H S(X)$.

Proof. Suppose $f$ is continuous, and let $h \in H S(X)$. Then, clearly, $\operatorname{int}_{H}(f(h)) \in \sigma$. Thus, by the hypothesis, $f^{-1}\left[\operatorname{int}_{H}(f(h))\right] \in \tau$. Since $f$ is injective, by Result $2(9), f^{-1}\left[\operatorname{int}_{H}(f(h))\right] \subset f^{-1}(f(h))=h$. Therefore, $f^{-1}\left[\operatorname{int}_{H}(f(h))\right] \subset \operatorname{int}_{H}(h)$. Hence, $\operatorname{int}_{H}(f(h)) \subset f\left(\operatorname{int}_{H}(h)\right)$.

From Theorem 11 and Proposition 22, we have the following result.

Corollary 2. Let $(X, \tau),(Y, \sigma)$ be hesitant fuzzy topological spaces. If $f:(X, \tau) \rightarrow(Y, \sigma)$ is continuous, open, and injective, then $\operatorname{int}_{H}(f(h))=f\left(\operatorname{int}_{H}(h)\right)$, for each $h \in H S(X)$.

Theorem 12. Let $(X, \tau),(Y, \sigma)$ be hesitant fuzzy topological spaces, and let $f: X \rightarrow Y$ be a mapping. Then, $f$ is closed if and only if $c l_{H}(f(h)) \subset f\left(c_{H}(h)\right)$, for each $h \in H S(X)$.

Proof. Suppose $f$ is closed, and let $h \in H S(X)$. Then, clearly, $h \subset c l_{H}(h)$. Thus, $f(h) \subset f\left(c l_{H}(h)\right)$. Since $f$ is closed and $c l_{H}(h)$ is closed in $X, f\left(c l_{H}(h)\right)$ is closed in Y. Therefore, $c l_{H}(f(h)) \subset f\left(c l_{H}(h)\right)$.

Conversely, assume the necessary condition holds, and let $F \in H F C(X)$. Then, clearly, $F=c l_{H}(F)$. Thus, by the hypothesis,

$$
c l_{H}(f(F)) \subset f\left(c l_{H}(F)\right)=f(F) \subset c l_{H}(f(F)) .
$$

Therefore, $c l_{H}(f(F))=f(F)$. Hence, $f(F)$ is closed in Y. Therefore, $f$ is closed.

The following is the immediate result of Theorems 10 and 12.

Corollary 3. Let $(X, \tau),(Y, \sigma)$ be hesitant fuzzy topological spaces. Then, a mapping $f:(X, \tau) \rightarrow(Y, \sigma)$ is continuous and closed if and only if $c l_{H}(f(F))=f\left(c l_{H}(h)\right)$, for each $h \in H S(X)$.

Definition 28. Let $(X, \tau),(Y, \sigma)$ be hesitant fuzzy topological spaces. Then, a mapping $f:(X, \tau) \rightarrow(Y, \sigma)$ is called a hesitant fuzzy homeomorphism, if it is bijective, continuous, and open.

Remark 3. For any hesitant fuzzy discrete spaces $X$ and $Y, f: X \rightarrow Y$ is a hesitant fuzzy homeomorphism if and only it is bijective.

\section{Hesitant Fuzzy Subspaces}

We define a hesitant fuzzy subspace topology, and we obtain some of its similar properties in classical topological spaces. Moreover, we prove that the "Pasting lemma" holds in hesitant fuzzy topological spaces. 
Definition 29. Let $(X, \tau)$ be a hesitant fuzzy topological space, and let $h \in H S(X)$. Then, the collection $\tau_{h}=\{U \widetilde{\cap} h: U \in \tau\}$ is called a hesitant fuzzy subspace topology or hesitant fuzzy relative topology on $h$. The pair $\left(h, \tau_{h}\right)$ is called a hesitant fuzzy subspace, and each member of $\tau_{h}$ is called a hesitant fuzzy open set in $h$.

Example 8. (1) Let $X=\{a, b, c\}$, and let $\tau=\left\{h^{0}, h^{1}, h_{1}, h_{2}, h_{3}, h_{4}\right\} \in \operatorname{HFT}(X)$, where $h_{1}(a)=\{0.4\} \cup[0.5,1), h_{1}(b)=(0.1,0.9], h_{1}(c)=[0.3,0.8) \cup\{0.1,0.9\}$,

$h_{2}(a)=(0.3,0.5) \cup[0.6,0.9], h_{2}(b)=\{0.2\} \cup(0.8,0.9), h_{2}(c)=(0.4,1] \cup\{0.1\}$,

$h_{3}(a)=(0.3,1), h_{3}(b)=(0.1,0.9], h_{3}(c)=[0.3,1] \cup\{0.1\}$,

$h_{4}(a)=\{0.4\} \cup[0.6,0.9], h_{4}(b)=\{0.2\} \cup(0.8,0.9), h_{4}(c)=(0.4,0.8) \cup\{0.1,0.9\}$.

Let $h$ be the hesitant fuzzy set in $X$ given by:

$h(a)=(0.4,0.9] \cup\{1\}, h(b)=[0.1,0.3) \cup[0.7,0.8], h(c)=[0.2,0.4) \cup(0.5,1]$.

Then, $\tau_{h}=\left\{h^{0}, h, h_{1} \widetilde{\cap} h, h_{2} \widetilde{\cap} h, h_{3} \widetilde{\cap} h, h_{4} \widetilde{\cap} h\right\}$.

(2) If $X$ is a hesitant fuzzy discrete space and $h \in H S(X)$, then $\left(h, \tau_{h}\right)$ is a hesitant fuzzy discrete space.

(3) If $X$ is a hesitant fuzzy indiscrete space and $h \in H S(X)$, then $\left(h, \tau_{h}\right)$ is a hesitant fuzzy indiscrete space.

Proposition 23. Let $(X, \tau)$ be a hesitant fuzzy topological space, and let $h \in H S(X)$. Then, $\tau_{h}$ is a hesitant fuzzy topology on $h$.

Proof. Since $h^{0}, h^{\mathbf{1}} \in \tau, h^{0}=h^{0} \widetilde{\cap} h \in \tau_{h}$ and $h=h^{\mathbf{1}} \widetilde{\cap} h \in \tau_{h}$.

Let $U, V \in \tau_{h}$. Then, there are $h_{1}, h_{2} \in \tau$ such that $U=h_{1} \widetilde{\cap} h$ and $V=h_{2} \widetilde{\cap} h$. Thus, $U \widetilde{\cap} V=$ $\left(h_{1} \widetilde{\cap} h\right) \widetilde{\cap}\left(h_{2} \widetilde{\cap} h\right)=\left(h_{1} \widetilde{\cap} h_{2}\right) \widetilde{\cap} h$. Since $h_{1} \widetilde{\cap} h_{2} \in \tau, U \widetilde{\cap} V \in \tau_{h}$.

Now, let $\left(U_{j}\right)_{j \in J} \subset \tau_{h}$. Then, there is $h_{j} \in \tau$ such that $U_{j}=h_{j} \widetilde{\cap} h$, for each $j \in J$. Thus, $\widetilde{U}_{j \in J} U_{j}=\widetilde{U}_{j \in J}\left(h_{j} \widetilde{\cap} h\right)=\left(\widetilde{U}_{j \in J} h_{j}\right) \widetilde{\cap} h$. Since $\widetilde{U}_{j \in J} h_{j} \in \tau, \widetilde{U}_{j \in J} U_{j} \in \tau_{h}$. Therefore, $\tau_{h}$ is a hesitant fuzzy topology on $h$.

Proposition 24. Let $(X, \tau)$ be a hesitant fuzzy topological space, and let $h_{1}, h_{2} \in H S(X)$ such that $h_{1} \subset h_{2}$. Then, $\tau_{h_{1}}=\left(\tau_{h_{2}}\right)_{h_{1}}$.

Proof. Let $U \in \tau_{h_{1}}$. Then, $V \in \tau$ such that $U=V \widetilde{\cap} h_{1}$. Since $h_{1} \subset h_{2}, h_{1}=h_{1} \widetilde{\cap} h_{2}$. Thus, $U=$ $V \widetilde{\cap}\left(h_{1} \widetilde{\cap} h_{2}\right)=\left(V \widetilde{\cap} h_{2}\right) \widetilde{\cap} h_{1}$. Since $V \widetilde{\cap} h_{2} \in \tau_{h_{2}}, U \in\left(\tau_{h_{2}}\right)_{h_{1}}$. Therefore, $\tau_{h_{1}} \subset\left(\tau_{h_{2}}\right)_{h_{1}}$. Let $U \in\left(\tau_{h_{2}}\right)_{h_{1}}$. Then, there is $V \in \tau_{h_{2}}$ such that $U=V \widetilde{\cap} h_{1}$. Since $V \in \tau_{h_{2}}$, there is $h \in \tau$ such that $V=h \widetilde{\cap} h_{2}$. Thus, $U=\left(h \widetilde{\cap} h_{2}\right) \widetilde{\cap} h_{1}=h \widetilde{\cap}\left(h_{2} \widetilde{\cap} h_{1}\right)$. Since $h_{1} \subset h_{2}, h_{2} \widetilde{\cap} h_{1}=h_{1}$. Therefore, $U=h \widetilde{\cap} h_{1}$. Hence, $U \in \tau_{h_{1}}$, i.e., $\left(\tau_{h_{2}}\right)_{h_{1}} \subset \tau_{h_{1}}$. Therefore, $\tau_{h_{1}}=\left(\tau_{h_{2}}\right)_{h_{1}}$.

Proposition 25. Let $(X, \tau)$ be a hesitant fuzzy topological space; let $h \in H S(X)$; and let $\mathcal{B}$ be a base for $\tau$. Then, $\mathcal{B}_{h}=\{B \widetilde{\cap} h: B \in \mathcal{B}\}$ is a base for $\tau_{h}$.

Proof. Let $U \in \tau$, and let $x_{\lambda} \in U \widetilde{\cap} h$. Then, there is $B \in \mathcal{B}$ such that $x_{\lambda} \in B \subset U$. Thus, $x_{\lambda} \in B \widetilde{\cap} h \subset$ $U \widetilde{n} h$. Therefore, by Proposition $4, \mathcal{B}_{h}$ is a base for $\tau_{h}$.

The following gives a special situation in which every member of the the hesitant fuzzy space topology is also a member of the hesitant topology on X.

Proposition 26. Let $(X, \tau)$ be a hesitant fuzzy topological space, and let $h \in \tau$. If $U \in \tau_{h}$, then $U \in \tau$.

Proof. Suppose $U \in \tau_{h}$. Then, there is $V \in \tau$ such that $U=V \widetilde{\cap} h$. Since $h \in \tau, V \widetilde{\cap} h \in \tau$. Thus, $U \in \tau$.

Theorem 13. Let $(X, \tau)$ be a hesitant fuzzy topological space; let $h \in H S(X)$; and $A \in H S(X)$ such that $A \subset h$. Then, $A$ is closed in $h$ if and only if there is $F \in H F C(X)$ such that $A=F \widetilde{\cap} h$. 
Proof. Assume $A$ is closed in $h$. Then clearly, $h \backslash A=h \widetilde{\cap} A^{c} \in \tau_{h}$. Thus, there is $U \in \tau$ such that $h \widetilde{\cap} A^{c}=U \widetilde{n} h$. Since $A \subset h, A=U^{c} \widetilde{\cap} h$. Let $F=U^{c}$. Since $U \in \tau, U^{c}$ is closed in $X$. Therefore, $F$ is closed in $X$. Hence, the necessary condition holds.

Conversely suppose the necessary condition holds, and let $A \in H S(X)$ such that $A \subset h$. Then, there is $F \in \operatorname{HFC}(X)$ such that $A=F \widetilde{\cap} h$. Thus, $F^{c} \in \tau$ and $h \backslash A=F^{c} \widetilde{\cap} h$. Therefore, $h \backslash A \in \tau_{h}$. Therefore, $A$ is closed in $h$.

There is also a criterion for a hesitant fuzzy closed set in a hesitant fuzzy subspace to be closed in the hesitant fuzzy topological space. The proof is similar to Proposition 26.

Proposition 27. Let $(X, \tau)$ be a hesitant fuzzy topological space, and let $h \in \operatorname{HFC}(X)$. If $A$ is closed in $\left(h, \tau_{h}\right)$, then $A \in \operatorname{HFC}(X)$.

Proposition 28. Let $(X, \tau)$ be a hesitant fuzzy topological space; let $h \in H F C(X)$; and let $A \subset h$. Then, $c l_{\tau_{h}}(A)=h \widetilde{\cap} c l_{H}(A)$, where $c l_{\tau_{h}}(A)$ denotes the closure of $A$ in $\left(h, \tau_{h}\right)$.

Proof. It is clear that $c l_{H}(A)$ is closed in $X$. Then, by Theorem $13, h \widetilde{\cap} c l_{H}(A)$ is closed in $\left(h, \tau_{h}\right)$. Since $A \subset h$ and $A \subset c l_{H}(A), A \subset h \widetilde{\cap} c l_{H}(A)$. Since:

$$
\operatorname{cl}_{\tau_{h}}(A)=\widetilde{\bigcap}\left\{F \in H S(X): A \subset F \text { and } F \text { is closed in }\left(h, \tau_{h}\right)\right\},
$$

$c l_{\tau_{h}}(A) \subset h \widetilde{\cap} c l_{H}(A)$. Since $c l_{\tau_{h}}(A)$ is closed in $\left(h, \tau_{h}\right)$, by Theorem 13, there is $F \in H F C(X)$ such that $c l_{\tau_{h}}(A)=h \widetilde{\cap} F$. Since $A \subset c l_{\tau_{h}}(A), A \subset F$. Since $F \in H F C(X), c l_{H}(A) \subset F$. Thus, $h \widetilde{\cap} c_{H}(A) \subset h \widetilde{\cap} F=$ $c l_{\tau_{h}}(A)$. Therefore, $c l_{\tau_{h}}(A)=h \widetilde{\cap} c l_{H}(A)$. This completes the proof.

From Definitions 13 and 29, we have the following result.

Theorem 14. Let $(X, \tau)$ be a hesitant fuzzy topological space; let $h \in H F C(X)$; let $N \subset h$; and let $a_{\lambda} \in h$. Then, $N \in \operatorname{HFN}_{\left(h, \tau_{h}\right)}\left(a_{\lambda}\right)$ if and only if there is $U \in \operatorname{HFN}\left(a_{\lambda}\right)$ such that $N=U \widetilde{n} h$, where $\operatorname{HFN}_{\left(h, \tau_{h}\right)}\left(a_{\lambda}\right)$ denotes the set of all neighborhoods of $a_{\lambda}$ in $\left(h, \tau_{h}\right)$.

Let $X$ be a nonempty set, and let $A$ be a subset of $X$. Then, we can consider $A$ as the mapping $A: X \rightarrow P[0,1]$ defined by: for each $x \in X$,

$$
A(x)=\left\{\begin{array}{lc}
{[0,1]} & \text { if } x \in A \\
\phi & \text { otherwise }
\end{array}\right.
$$

In this case, $A$ is also a hesitant fuzzy set, and we will write $A$ as $A=h_{A}^{\mathbf{1}}$.

Remark 4. Let $(X, \tau)$ be a hesitant fuzzy topological space, and let $A \subset X$. Then, we can easily see that the collection $\tau_{h_{A}^{1}}=\left\{U \widetilde{\cap} h_{A}^{1}: U \in \tau\right\}$ is a hesitant fuzzy subspace topology on A. Furthermore, we can see that all the propositions and all theorems obtained in the above hold in $\left(A, \tau_{h_{A}^{1}}\right)$.

Proposition 29. Let $(X, \tau),(Y, \sigma)$ be hesitant fuzzy topological spaces, and let $A \subset X, B \subset Y$.

(1) The inclusion mapping $i:\left(A, \tau_{h_{A}^{1}}\right) \rightarrow(X, \tau)$ is continuous.

(2) If $f:(X, \tau) \rightarrow(Y, \sigma)$ is continuous, then $\left.f\right|_{A}:\left(A, \tau_{h_{A}^{1}}\right) \rightarrow(Y, \sigma)$ is continuous.

(3) If $f:(X, \tau) \rightarrow\left(B, \sigma_{h_{B}^{1}}\right)$ is continuous, then the mapping $g:(X, \tau) \rightarrow(Y, \sigma)$ defined by $g(x)=f(x)$ for each $x \in X$ is continuous.

(4) If $f:(X, \tau) \rightarrow(Y, \sigma)$ and $f(X) \subset B$, then the mapping $g:(X, \tau) \rightarrow\left(B, \sigma_{h_{B}^{1}}\right)$ defined by $g(x)=f(x)$ for each $x \in X$ is continuous.

Proof. (1) Let $V \in \tau$. Then, clearly, $i^{-1}(V)=V \widetilde{\cap} h_{A}^{1}$. Thus, $i^{-1}(V) \in \tau_{h_{A}^{1}}$. Therefore, $i$ is continuous. 
(2) Let $V \in \sigma$. Then clearly, $\left(\left.f\right|_{A}\right)^{-1}(V)=f^{-1}(V) \widetilde{n} h_{A}^{1}$. Since $f$ is continuous, $f^{-1}(V) \in \tau$. Thus, $\left(\left.f\right|_{A}\right)^{-1}(V) \in \tau_{h_{A}^{1}}$. Therefore, $\left.f\right|_{A}$ is continuous.

(3) Let $V \in \sigma$. Then clearly, $V \widetilde{\cap} h_{B}^{1} \in \sigma_{h_{B}^{1}}$. Since $f$ is continuous,

$$
f^{-1}\left(V \widetilde{\cap} h_{B}^{\mathbf{1}}\right)=f^{-1}(V) \widetilde{\cap} f^{-1}\left(h_{B}^{\mathbf{1}}\right)=f^{-1}(V) \widetilde{\cap} h_{X}^{1}=f^{-1}(V) \in \tau .
$$

Thus, by the definition of $g, g^{-1}(V)=f^{-1}(V)$. Therefore, $g^{-1}(V) \in \tau$. Hence, $g$ is continuous.

(4) Suppose $f:(X, \tau) \rightarrow(Y, \sigma)$ and $f(X) \subset B$. Let $V \in \sigma_{h_{B}^{1}}$. Then, there is $U \in \sigma$ such that $V=U \widetilde{\cap} h_{B}^{1}$. Thus, by the hypothesis, $f^{-1}(U) \in \tau$. Since $f(X) \subset B$, by the definition of $g$,

$$
g^{-1}(V)=g^{-1}\left(U \widetilde{\cap} h_{B}^{\mathbf{1}}\right)=g^{-1}(U) \widetilde{\cap} g^{-1}\left(h_{B}^{\mathbf{1}}\right)=f^{-1}(U) \widetilde{\cap} h_{X}^{\mathbf{1}}=f^{-1}(U) .
$$

Therefore, $g^{-1}(V) \in \tau$. Hence, $g$ is continuous.

Proposition 30. Let $(X, \tau),(Y, \sigma)$ be hesitant fuzzy topological spaces, and let $f: X \rightarrow Y$ be a mapping. Let $\left(U_{j}\right)_{j \in J}$ be any family of subsets of $X$ such that $X=\bigcup_{j} U_{j}$, and let $\left(U_{j}\right)_{j \in J} \subset \tau$. If $\left.f\right|_{U_{j}}:\left(U_{j}, \tau_{h_{U_{j}}^{1}}\right) \rightarrow(Y, \sigma)$ is continuous, for each $j \in J$, then $f:(X, \tau) \rightarrow(Y, \sigma)$ is continuous.

Proof. Let $V \in \sigma$. Then, by the hypothesis, $\left(f \mid U_{j}\right)^{-1}(V) \in \tau_{h_{u_{j}}^{1}}$. Since $U_{j} \in \tau$, by Proposition 26, $\left(\left.f\right|_{U_{j}}\right)^{-1}(V) \in \tau$. On the other hand, $f^{-1}(V)=\widetilde{U}_{j \in J}\left(\left.f\right|_{U_{j}}\right)^{-1}(V)$. Thus, $f^{-1}(V) \in \tau$. Therefore, $f$ is continuous.

Proposition 31. (Pasting lemma). Let $(X, \tau),(Y, \sigma)$ be hesitant fuzzy topological spaces, and let $A, B \subset X$ such that $X=A \cup B$ and $A, B \in \operatorname{HFC}(X)$. Let $f:\left(A, \tau_{h_{A}^{1}}\right) \rightarrow(Y, \sigma)$ and $g:\left(B, \tau_{h_{B}^{1}}\right) \rightarrow(Y, \sigma)$ be continuous mappings such that $f(x)=g(x)$ for each $x \in A \cap B$. Then, the mapping $h:(X, \tau) \rightarrow(Y, \sigma)$ defined by $h(x)=f(x)$ for each $x \in A$ and $h(x)=g(x)$ for each $x \in B$ is continuous.

Proof. Let $F \in H F C(Y)$. Since $f$ and $g$ are continuous, by Theorem $10, f^{-1}(F)$ is closed in $\left(A, \tau_{h_{A}^{1}}\right)$, and $g^{-1}(F)$ is closed in $\left(B, \tau_{h_{B}^{1}}\right)$. Since $A, B \in H F C(X)$, by Proposition $26, f^{-1}(F), g^{-1}(F) \in \operatorname{HFC}(X)$. Then, $h^{-1}(F)=f^{-1}(F) \widetilde{\cap} g^{-1}(F) \in H F C(X)$. Thus, by Theorem 10, $h$ is continuous.

\section{Hesitant Fuzzy Product Topologies and Initial Topologies}

We define a hesitant fuzzy product topology and prove that there exists an initial structure in hesitant fuzzy topological spaces (See Theorem 16).

Definition 30. Let $\left(X_{j}, \tau_{j}\right)_{j \in J}$ be a family of hesitant fuzzy topological spaces; let $X=\prod_{j \in J} X_{j}$; and let $\left(\pi_{j}: X \rightarrow X_{j}\right)_{j \in J}$ be a family of projections. For each $j \in J$, let $\mathcal{S}_{j}=\left\{\pi_{j}^{-1}(U): U \in \tau_{j}\right\}$, and let $\mathcal{S}=\bigcup_{j \in J} \mathcal{S}_{j}$. Then, $\mathcal{S}$ is a sub-base for a hesitant fuzzy topology $\tau$ on $X$ induced by $\left(\pi_{j}: X \rightarrow\left(X_{j}, \tau_{j}\right)\right)_{j \in J}$.

In this case, $\tau$ is called the hesitant fuzzy product topology on $X$ and will be denoted by $\prod_{j \in J} \tau_{j}$. The pair $(X, \tau)$ is called a hesitant fuzzy product space.

Proposition 32. Let $\left(X_{j}, \tau_{j}\right)_{j \in J}$ be a family of hesitant fuzzy topological spaces. Then, the hesitant fuzzy product topology $\prod_{j \in J} \tau_{j}$ is the coarsest hesitant fuzzy topology on $\prod_{j \in J} X_{j}$ for which each $\pi_{i}:\left(\prod_{j \in J} X_{j}, \prod_{j \in J} \tau_{j}\right) \rightarrow$ $\left(X_{i}, \tau_{i}\right)$ is continuous.

Proof. Let $\mathcal{S}$ be the sub-base for the hesitant fuzzy product topology $\prod_{j \in J} \tau_{j}$. For each $i \in J$, let $U \in \tau_{i}$. Then clearly, $\pi_{i}^{-1}(U) \in \mathcal{S}$. Since $\mathcal{S} \subset \prod_{j \in J} \tau_{j}, \pi_{i}^{-1}(U) \in \prod_{j \in J} \tau_{j}$. Thus, by Theorem 10 (3), $\pi_{i}$ is continuous, for each $i \in J$. 
Suppose $\tau$ is any hesitant fuzzy topology on $\prod_{j \in J} X_{j}$ for which each $\pi_{i}$ is continuous, where $\pi_{i}:\left(\prod_{j \in J} X_{j}, \prod_{j \in J} \tau_{j}\right) \rightarrow\left(X_{i}, \tau_{i}\right)$ is the projection mapping. Let $S \in \mathcal{S} \subset \prod_{j \in J} \tau_{j}$. Then, there are $j \in J$ and $U \in \tau_{j}$ such that $S=\pi_{j}^{-1}(U)$. Thus, by the hypothesis, $\pi_{j}^{-1}(U) \in \tau$, i.e., $S \in \tau$. Therefore, $\prod_{j \in J} \tau_{j} \subset \tau$.

Theorem 15. Let $\left(Y_{j}, \tau_{j}\right)_{j \in J}$ be a family of hesitant fuzzy topological spaces, and let $(X, \tau)$ be a hesitant fuzzy topological space. Then, a mapping $f:(X, \tau) \rightarrow\left(\prod_{j \in J} Y_{j}, \prod_{j \in J} \tau_{j}\right)$ is continuous if and only if $\pi_{j} \circ f:(X, \tau) \rightarrow\left(Y_{j}, \tau_{j}\right)$ is continuous, for each $j \in J$.

Proof. Suppose $f$ is continuous, and let $j \in J$. Then, by Proposition $32, \pi_{j}$ is continuous, where $\pi_{j}$ : $\left(\prod_{j \in J} Y_{j}, \prod_{j \in J} \tau_{j}\right) \rightarrow\left(Y_{j}, \tau_{j}\right)$ is the projection mapping. Thus, by Proposition 20, $\pi_{j} \circ f$ is continuous.

Conversely, suppose the necessary condition holds, and let $\mathcal{S}$ be the sub-base for the hesitant fuzzy product topology $\prod_{j \in J} \tau_{j}$ on $\prod_{j \in J} Y_{j}$ given by Definition 30. For each $j \in J$ and each $U \in \tau_{j}$, let $\pi_{j}^{-1}(U) \in \mathcal{S}$. Then, $f^{-1}\left(\pi_{j}^{-1}(U)\right)=\left(\pi_{j} \circ f\right)^{-1}(U)$. Thus, by the hypothesis, $\left(\pi_{j} \circ f\right)^{-1}(U) \in \tau$. Therefore, $f^{-1}\left(\pi_{j}^{-1}(U)\right) \in \tau$. Hence, by Theorem 10 (3), $f$ is continuous.

The following is the immediate result of Theorem 15.

Corollary 4. Let $\left(Y_{j}, \tau_{j}\right)_{j \in J}$ be a family of hesitant fuzzy topological spaces; let $(X, \tau)$ be a hesitant fuzzy topological space and for each $j \in J$; let $f_{j}: X \rightarrow Y_{j}$ be a mapping. We define a mapping $f: X \rightarrow \prod_{j \in J} Y_{j}$ as follows:

$$
\text { for each } x \in X \text { and each } j \in J \text {, the value of } f(x) \text { at } j \text { is } f_{j}(x) \text {. }
$$

Then, $f$ continuous if and only if $f_{j}$ is continuous, for each $j \in J$.

Proposition 32 is the motivation of the following definition.

Definition 31. Let $X$ be a nonempty set; let $\left(X_{j}, \tau_{j}\right)_{j \in J}$ be a family of hesitant fuzzy topological spaces; and let $\left(f_{j}: X \rightarrow\left(X_{j}, \tau_{j}\right)\right)_{j \in J}$ be a family of mappings. For each $j \in J$, let $\mathcal{S}_{j}=\left\{f_{j}^{-1}(U): U \in \tau_{j}\right\}$, and let $\mathcal{S}=\cup_{j \in J} \mathcal{S}_{j}$. Then, the coarsest hesitant fuzzy topology $\tau$ on $X$ with the sub-base $\mathcal{S}$ for which each $f_{j}:(X, \tau) \rightarrow\left(X_{j}, \tau_{j}\right)$ is continuous.

Especially, $\tau$ is called the hesitant fuzzy initial (or weak) topology on $X$ induced by $\left(f_{j}: X \rightarrow\left(X_{j}, \tau_{j}\right)\right)_{j \in J}$.

From Proposition 32, we can easily see that the hesitant fuzzy product topology $\prod_{j \in J} \tau_{j}$ on $\prod_{j \in J} X_{j}$ is the hesitant fuzzy initial topology induced by the family $\left(\pi_{j}: \prod_{j \in J} X_{j} \rightarrow X_{j}\right)_{j \in J}$ of projection mappings. By Theorem 15, we obtain the following theorem.

Theorem 16. Let $\left(X_{j}, \tau_{j}\right)_{j \in J}$ be a family of hesitant fuzzy topological spaces; let $x$ be a set and for each $j \in J$; let $f_{j}: X \rightarrow\left(X_{j}, \tau_{j}\right)$ be a mapping. Let $\tau$ be the hesitant fuzzy initial topology on $X$ with the sub-base $\mathcal{S}$ induced by $\left(f_{j}: X \rightarrow\left(X_{j}, \tau_{j}\right)\right)_{h \in J}$, where $\mathcal{S}=\left\{f_{j}^{-1}(U): U \in \tau_{j}, j \in J\right\}$. Let $(Y, \sigma)$ be a hesitant fuzzy topological space. Then, a mapping $f:(Y, \sigma) \rightarrow(X, \tau)$ is continuous if and only if $f_{j} \circ f:(Y, \sigma) \rightarrow\left(X_{j}, \tau_{j}\right)$ is continuous, for each $j \in J$.

Proof. Suppose $f$ is continuous, and let $j \in J$. Then, by the definition of the hesitant fuzzy initial topology, $f_{j}:(X, \tau) \rightarrow\left(Y_{j}, \tau_{j}\right)$ is continuous. Thus, by Proposition 20, $f_{j} \circ f$ is continuous.

Conversely, suppose the necessary condition holds, and let $\mathcal{S}$ be the sub-base for the hesitant fuzzy initial topology $\tau$ on $X$ given by Definition 31. For each $j \in J$ and each $U \in \tau_{j}$, let $f_{j}^{-1}(U) \in$ $\mathcal{S}$. Then, $f^{-1}\left(f_{j}^{-1}(U)\right)=\left(f_{j} \circ f\right)^{-1}(U)$. Thus, by the hypothesis, $\left(f_{j} \circ f\right)^{-1}(U) \in \tau$. Therefore, $f^{-1}\left(f_{j}^{-1}(U)\right) \in \tau$. Hence, by Theorem 10 (3), $f$ is continuous. 
Proposition 33. Let $\left(X_{j}, \tau_{j}\right)_{j \in J}$ be a family of hesitant fuzzy topological spaces; let $X$ be a set and for each $j \in J$; let $f_{j}: X \rightarrow\left(X_{j}, \tau_{j}\right)$ be a mapping. Let $\tau$ be the hesitant fuzzy initial topology on $X$ with the sub-base $\mathcal{S}$ induced by $\left(f_{j}: X \rightarrow\left(X_{j}, \tau_{j}\right)\right)_{j \in J}$, and let $A \subset X$. Then, $\tau_{h_{A}^{1}}$ is the hesitant fuzzy initial topology on $A$ induced by $\left(f_{j} \mid A\right)_{j \in J}$.

Proof. Let $\delta$ be the hesitant fuzzy initial topology on $A$ induced by $\left(\left.f_{j}\right|_{A}\right)_{j \in J}$, and let $\mathcal{S}^{\prime}=\left\{\left(\left.f_{j}\right|_{A}\right.\right.$ )$\left.^{-1}\left(U_{j}\right): U_{j} \in \tau_{j}, j \in J\right\}$. Then, clearly, $\mathcal{S}^{\prime}$ is a sub-base for $\delta$. In order to prove that $\delta \subset \tau_{h_{A}^{1}}$, for each $j \in J$ and $U_{j} \in \tau_{j}$, let $\left(\left.f_{j}\right|_{A}\right)^{-1}\left(U_{j}\right) \in \mathcal{S}^{\prime}$. Then, clearly, $\left(\left.f_{j}\right|_{A}\right)^{-1}\left(U_{j}\right)=f_{j}^{-1}\left(U_{j}\right) \widetilde{\cap} h_{A}^{\mathbf{1}}$. Since $\tau$ is the hesitant fuzzy initial topology on $X$ induced by $\left(f_{j}: X \rightarrow\left(Y_{j}, \tau_{j}\right)\right)_{h \in J}, f_{j}^{-1}\left(U_{j} \in \tau\right.$, thus, by Remark 4 , $f_{j}^{-1}\left(U_{j}\right) \widetilde{\cap} h_{A}^{\mathbf{1}} \in \tau_{h_{A}^{1}}$. Therefore, $\left(\left.f_{j}\right|_{A}\right)^{-1}\left(U_{j}\right) \in \tau_{h_{A}^{1}}$. Hence, $\delta \subset \tau_{h_{A}^{1}}$.

Now, let us show that $\tau_{h_{A}^{1}} \subset \delta$. For each $j \in J$ and $U_{j} \in \tau_{j}$, let $f_{j}^{-1}\left(U_{j}\right) \in \mathcal{S} \subset \tau$. Then, clearly, $f_{j}^{-1}\left(U_{j}\right) \widetilde{\cap} h_{A}^{\mathbf{1}} \in \delta$ and $f_{j}^{-1}\left(U_{j}\right) \widetilde{\cap} h_{A}^{\mathbf{1}}=\left(\left.f_{j}\right|_{A}\right)^{-1}\left(U_{j}\right)$. Thus, $\left(\left.f_{j}\right|_{A}\right)^{-1}\left(U_{j}\right) \in \delta$. Therefore, $\tau_{h_{A}^{1}} \subset \delta$. This completes the proof.

\section{Conclusions}

We defined a hesitant fuzzy topology, a hesitant fuzzy base and sub-base, a hesitant fuzzy neighborhood and Q-neighborhood, a hesitant fuzzy closure and interior, a hesitant fuzzy continuous mapping, a hesitant fuzzy subspace, and a hesitant fuzzy product space, and obtained some of their properties, respectively. In the future, we will try to separate the axioms, compactness, and connectedness in hesitant fuzzy topological space.

Author Contributions: This paper was organized by the idea of K.H., J.-G.L. analyzed the related papers with this research, and they also wrote the paper. Conceptualization, J.-G.L.; Writing-review \& editing, K.H. All authors have contributed equally to this paper in all aspects. All authors have read and agreed to the published version of the manuscript.

Acknowledgments: This paper was supported by Wonkwang University in 2019.

Conflicts of Interest: The authors declare no conflict of interest.

\section{References}

1. Zadeh, L.A. Fuzzy sets. Inf. Control. 1965, 8, 338-353. [CrossRef]

2. Chang, C.L. Fuzzy topological spaces. J. Math. Anal. Appl. 1968, 24, 182-190. [CrossRef]

3. Ekici, E. More on $\theta$-compact fuzzy topological spaces. Chaos. Solitons Fractals 2006, 27, 1151-11161. [CrossRef]

4. Silva, C.A.S.; Landim, R.R. Fuzzy spaces topology change and BH thermodynamics. J. Phys. Conf. Ser. 2014, 490, 012012. [CrossRef]

5. Gantner, T.E.; Steinlage, R.C.; Waren, R.H. Compactness in fuzzy topological spaces. J. Math. Anal. Appl. 1978, 62, 547-562. [CrossRef]

6. Guojun, W. A new fuzzy compactness defined by fuzzy nets. J. Math. Anal. Appl. 1983, 94, 1-23. [CrossRef]

7. Lowen, R. Fuzzy topological spaces and fuzzy compactness. J. Math. Anal. Appl. 1976, 56, 621-633. [CrossRef]

8. Lowen, R. Initial and final fuzzy topologies and the fuzzy Tychonoff theorem. J. Math. Anal. Appl. 1977, 58, 11-21. [CrossRef]

9. Lowen, R. A comparison of different compactness notions in fuzzy topological spaces. J. Math. Anal. Appl. 1978, 64, 446-454. [CrossRef]

10. Pu, P.M.; Liu, Y.M. Fuzzy topology I: Neighborhood structure of a fuzzy point. J. Math. Anal. Appl. 1980, 76, 571-599.

11. Pu, P.M.; Liu, Y.M. Fuzzy topology II: Products and quotient spaces. J. Math. Anal. Appl. 1982, 77, $20-37$.

12. Sinha, S.P. Separation axioms in fuzzy topological spaces. Fuzzy Sets Syst. 1992, 45, 261-270. [CrossRef]

13. Torra, V. Hesitant fuzzy sets. Int. J. Intell. Syst. 2010, 25, 529-539. [CrossRef]

14. Pei, Z.; Yi, L. A note on operations of hesitant fuzzy set. Int. J. Comput. Intell. Syst. 2015, 8, $226-239$. [CrossRef] 
15. Thakur, G.S.; Thakur, R.; Singh, R. New Hesitant fuzzy operatos. Fuzzy Inf. Eng. 2014, 6, 379-392. [CrossRef]

16. Xia, M.; Xu, Z. Hesitant fuzzy information aggregation in decision making. Int. J. Approx. Reason. 2011, 52, 395-407. [CrossRef]

17. Jun, Y.B.; Lee, K.J.; Song, S.Z. Hesitant fuzzy bi-ideals in semigroups. Commun. Korean Math. Soc. 2015, 30, 143-154. [CrossRef]

18. Divakaran, D.; John, S.J. Hesitant fuzzy rough sets through hesitant fuzzy relations. Ann. Fuzzy Math. Inform. 2014, 8, 33-46.

19. Divakaran, D.; John, S.J. Homomorphism of hesitant fuzzy subgroups. Int. J. Sci. Eng. Res. 2014, 5, 9-14.

20. Divakaran, D.; John, S.J. Hesitant fuzzy subgroups. J. New Theory 2016, 11, 54-68.

21. Divakaran, D.; John, S.J. Dual hesitant fuzzy subrings and ideals. Ann. Fuzzy Math. Inform. 2017, 13, 437-448.

22. Jun, Y.B.; Ahn, S.S. Hesitant fuzzy sets theory applied to BCK/BCI-algebras. J. Comput. Anal. Appl. 2016, 20, 635-646.

23. Kim, J.; Lim, P.K.; Lee, J.G.; Hur, K. Hesitant fuzzy sets applied to BCK/BCI-algebras. Ann. Fuzzy Math. Inform. 2019, 18, 209-231. [CrossRef]

24. Kim, J.; Jun, Y.B.; Lim, P.K.; Lee, J.G.; Hur, K. The category of hesitant H-fuzzy sets. Ann. Fuzzy Math. Inform. 2019, 18, 57-74. [CrossRef]

25. Deepak, D.; Mathew, B.; John, S.J.; Garg, H. A topological structure involving hesitant fuzzy sets. J. Intell. Fuzzy Syst. 2019, 36, 6401-6412. [CrossRef]

26. Kim, J.; Lim, P.K.; Lee, J.G.; Hur, K. Hesitant fuzzy subgroups and subrings. Ann. Fuzzy Math. Inform. 2019, 18, 105-122. [CrossRef]

27. Patty, C.W. Foundations of Topology; PWS-KENT Publishing Co.: Boston, MA, USA, 1993.

28. Pervin, W.J. Foundations of General Topology; Academic Press Inc.: New York, NY, USA, 1964.

(C) 2020 by the authors. Licensee MDPI, Basel, Switzerland. This article is an open access article distributed under the terms and conditions of the Creative Commons Attribution (CC BY) license (http://creativecommons.org/licenses/by/4.0/). 